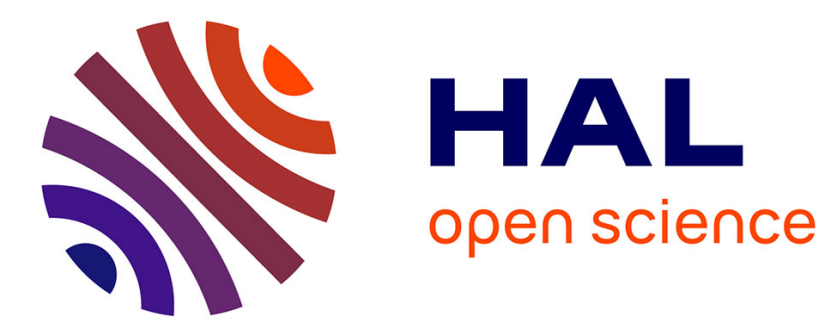

\title{
La Préhistoire ancienne en Gironde: apport des recherches récentes
}

Michel Lenoir

\section{To cite this version:}

Michel Lenoir. La Préhistoire ancienne en Gironde: apport des recherches récentes. Gallia Préhistoire - Archéologie de la France préhistorique, 2000, 42, pp.57-84. 10.3406/galip.2000.2170 hal-02353530

\section{HAL Id: hal-02353530 \\ https://hal.science/hal-02353530}

Submitted on 21 Jan 2020

HAL is a multi-disciplinary open access archive for the deposit and dissemination of scientific research documents, whether they are published or not. The documents may come from teaching and research institutions in France or abroad, or from public or private research centers.
L'archive ouverte pluridisciplinaire HAL, est destinée au dépôt et à la diffusion de documents scientifiques de niveau recherche, publiés ou non, émanant des établissements d'enseignement et de recherche français ou étrangers, des laboratoires publics ou privés.

\section{(이) $\$$}

Distributed under a Creative Commons Attribution - NonCommercial - NoDerivatives| 4.0 


\title{
La Préhistoire ancienne en Gironde
}

\author{
Apports des recherches récentes
}

\author{
Michel LENOIR*
}

Mots-clés. Gironde, Dordogne, Garonne, Paléolithique, Magdalénien, industries, technocomplexes, cultures matérielles, temps glaciaires.

Key-words. Gironde, Dordogne, Garonne, Pulueolithic, Magdalenian, industries, technocomplexes, material cultures, glacial times.

Résumé. Dans les basses vallées de la Dordogne et de la Garonne, les indices les plus anciens de présence humaine remontent au Mindel. À partir du Riss cette présence de l'homme est manifeste et s'affirme dans des sites de plein air implantés le plus souvent sur des gites de silex lacustre ou repris dans les alluvions rissiennes. Au cours du Würm ancien, l'habitat demeure presque exclusivement de plein air excepté pour les couches profondes de la grotte de Pair-non-Pair. Le Châtelperronien, bien que représenté dans ce secteur, y demeure discret, dans des cavités qui ont également servi de repaires d'hyènes. Aurignaciens et Gravettiens ont fréquenté des habitats sporadiques de plein air et quelques cavités qui ont livré des vestiges lithiques et osseux. Longtemps contestée, la présence de Solutréen est désormais bien attestée au pied de la falaise du Grand-Moulin, dans l'Entre-Deux-Mers, outre quelques indices sporadiques en plein air. Les Badegouliens se sont généralement établis en plein air sur des points hauts, plus rarement en pied de falaise et y ont abandonné des industries polymorphes. Magdaléniens et Aziliens occupent des cavités, grottes et abris sous roche, des sites en pied de falaise et des campements de plein air sur des versants placés en contrebas de lignes de falaises ou sur des plateaux sableux dans le cas d'un gisement actuellement en cours de fouille dans la haute lande girondine, entre la Garonne et l'océan. Deux gisements, Saint-Germain-la-Rivière et le Roc de Marcamps, qui ont livré des séquences représenlatives de Magdalénien ancien et moyen, ont fait l'objet de travaux récents et le Magdalénien supérieur connu dans quelques gisements sous abri montre l'apparition d'armatures nouvelles.

Abstract. In the low Dordogne and Garonne valleys the oldest prehistoric occupation appears during the Mindel but the human presence becomes more obvious during the Riss, in numerous open air settlements close to local flint sources or mixed assemblages in the alluvial deposits. During the old Würm the Mousterian people almost exclusively settle in open air stations, less frequently in caves like Pair-nonPair's lowest layers. In this geographical area the Chatelperronian remains discrete. Aurignacians and Gravettians settled in open air sites, on foot-clives or in small rockshelters with lithic assemblages and bones. The long controversy on a Solutrean occurrence on the footcliff of Grand-Moulin is nowadays closed. Badegoulian occupation is usually known in small open air sites, on top hills, more scarcely on footclives, by polymorphous lithic industries. Magdalenian sites are numerous, in rockshelters along some small tributaries of the Dordogne valley, sometimes on open air slopes close to limestome clives and in one case, actually dug, in the sandy plateau between the river Garonne and the atlantic coast. Two main sites, with representative sequences of lower and middle Magdalenian have been recently revised and the Upper Magdalenian known in small rockshelters, shows the invention of new projectile points.

\section{PRÉSENTATION GÉNÉRALE}

Les basses vallées de la Dordogne et de la Garonne fusionnent en un vaste estuaire qui se jette dans l'océan.
Ce secteur appartient à l'Aquitaine occidentale. Il s'ouvre sur la façade atlantique et se place dans un bassin sédimentaire où les potentialités de subsistance pour l'homme paléolithique sont nombreuses et variées, qu'il

* Institut de Préhistoire et de Géologie du Quaternaire, UMR 5808 du CNRS, Université Bordeaux I, bâtiment de géologie, avenue des Facultés, F-33405 Talence Cedex. 


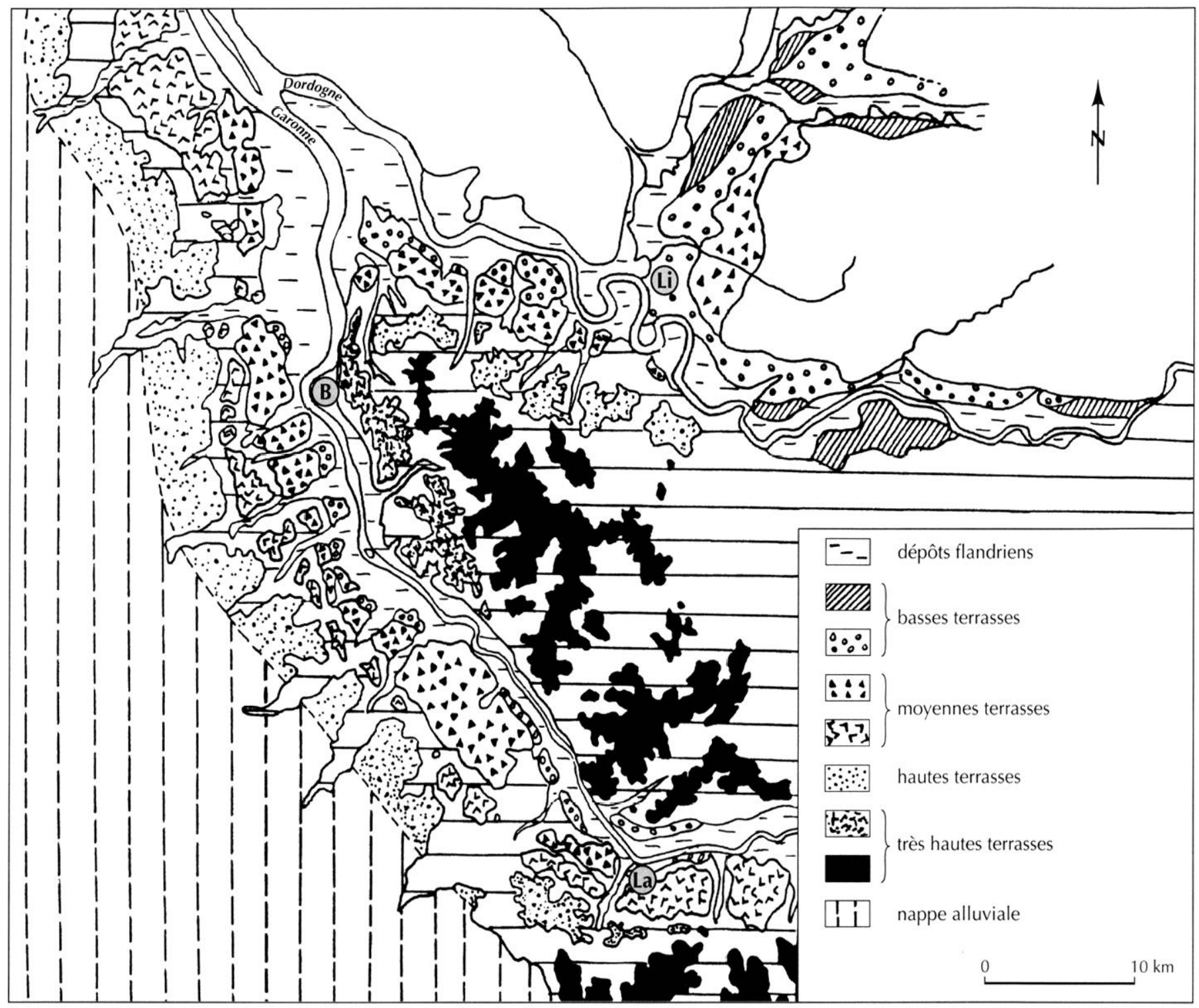

Fig. 1 - Répartition des dépôts quaternaires dans les basses vallées de la Dordogne el de la Garonne (d'après J. Dubreuilh, 1976 et L. Moisan, 1978a, 1978b).

s'agisse des ressources en matières premières lithiques, du gibier, des ressources végétales. Ce secteur des basses vallées de la Dordogne et de la Garonne est de faible altitude, relativement plat, de topographie régulière, accidentée dans la partie interne calcaire de quelques buttes résiduelles de calcaire lacustre, et recoupé par un réseau hydrographique assez dense. Les deux axes fluviaux, Dordogne et Garonne et leurs affluents majeurs tels que I'Isle pour la Dordogne, le Dropt pour la Garonne, ont pu servir de voies de passage. Il en est de même de certains affluents qui dans le secteur calcaire karstique de
l'Entre-Deux-Mers, entre Dordogne et Garonne, sont bordés de lignes de falaises creusées de cavités aujourd'hui pour la plupart effondrées et dont certaines ont été occupées par l'homme préhistorique. En outre, lors des périodes les plus froides du Pléistocène, le plateau continental devait être largement dégagé et il a donc pu être parcouru, voire même habité, par les Paléolithiques.

Dans ce secteur trois entités majeures peuvent être distinguées.

Les plateaux en rive droite de la Dordogne, limités par des lignes de falaises plus ou moins hautes et plus ou 
moins dégagées, dominent le fleuve et l'estuaire et sont localement interrompus par le débouché de vallées. Le calcaire marin stampien à Astéries représenté dans la partie ouest y cède la place à l'est au calcaire lacustre de Castillon. Ces plateaux sont coiffés par des dépôts de revêtement limono-sableux ou argileux ou par de hautes terrasses alluviales.

Au centre, entre Dordogne et Garonne, s'étend l'Entre-Deux-Mers, vaste plateau dont le calcaire à Astéries constitue l'assise principale qui repose localement sur le calcaire et les argiles de Castillon, tandis que les calcaires lacustres aquitaniens ne subsistent que sous forme de lambeaux au faîte de buttes témoins. Le réseau hydrographique entaille ces divers bancs rocheux et fait affleurer, sur les versants des vallées, des dépôts argilomolassiques. La molasse de l'Agenais culmine par endroits au toit des dépôts oligo-miocènes. Ce substratum est masqué par des dépôts pléistocènes: hautes nappes alluviales résiduelles, limons de revêtement, dépôts de pente et de versants, alluvions de fond de vallées. Le cours de la Garonne suit une ligne de faille qui sépare deux compartiments décalés en altitude, le plateau de l'Entre-Deux-Mers à l'est, le pays de Graves et la lande girondine à l'ouest.

A l'ouest de la Garonne (fig. 1), entre fleuve et océan, les terrasses alluviales du Médoc, des Graves et du Sauternais cèdent la place en direction de l'ouest à des formations superficielles sablo-graveleuses ainsi qu'à la couverture sableuse d'origine éolienne le plus souvent reprise par ruissellement. Le calcaire stampien et les faluns miocènes affleurent en fond de vallées dans le secteur nord-occidental nappé de terrasses alluviales et de sables éoliens, tandis qu'au sud-est, dans le Bazadais, des bancs de grès calcaires et de calcaires aquitaniens coiffent d'épaisses formations argileuses miocènes. Ces formations, entaillées par de profonds vallons, donnent une topographie plus accidentée. Dans la lande girondine, un bombement anticlinal (ride de Villagrains-Landiras) fait affleurer des calcaires campaniens à nodules de silex. D'autres anticlinaux dans le Médoc et le Blayais font apparaître des formations éocènes.

La forêt de pins des Landes se développe vers l'ouest sur les nappes sablo-graveleuses émaillées par endroits de dunes anciennes, elles-mêmes relayées vers le littoral par des cordons de dunes récentes. Une ligne d'étangs s'échelonne du Médoc au Pays basque entre le littoral et le massif forestier.

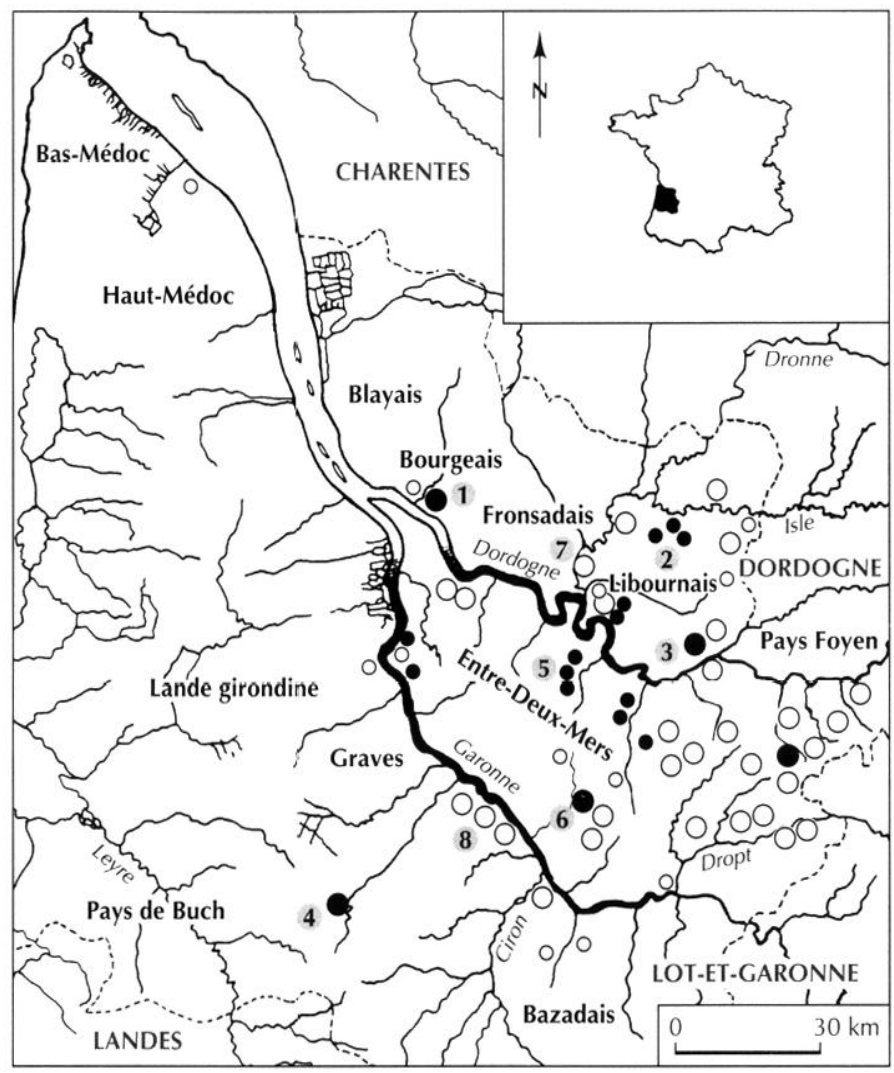

Fig. 2 - Densité de répartition des principaux sites du Paléolithique ancien et moyen des basses vallées de la Dordogne et de la Garonne (O Paléolithique ancien, Paléolithique moyen) : 1, Pair-non-Pair;

2, Cornemps et Puynormand ; 3, Chinchon ; 4, Villagrains ;

5, Camiac; 6, Haurets; 7, terrasses alluviales du Libournais ; 8, terrasses alluviales des Graves.

\section{LES PLUS ANCIENNES OCCUPATIONS}

Les industries préhistoriques les plus anciennes pour l'instant connues en Gironde ont été découvertes en contexte alluvial mindélien dans les basses vallées de l'Isle, de la Dordogne (Moisan, 1978a, 1978b, 1979, 1986, 1987) et de la Garonne (Millet, 1991, 1992; Millet, Millet, 1991), outre un fragment crânien trouvé hors stratigraphie mais qui pourrait être mindélien (Bouvier, Rousseau, 1972). Quelques vestiges lithique non roulés (8 éclats et 2 nucléus) ont été recueillis groupés, par L. Moisan, sous une formation rubéfiée reposant sur un paléosol qui pourrait dater de l'interglaciaire GünzMindel ou d'un interstade mindélien (Moisan, 1978a, 1978b).

L'Acheuléen est abondant dans les moyennes terrasses alluviales des basses vallées de l'Isle et de la 


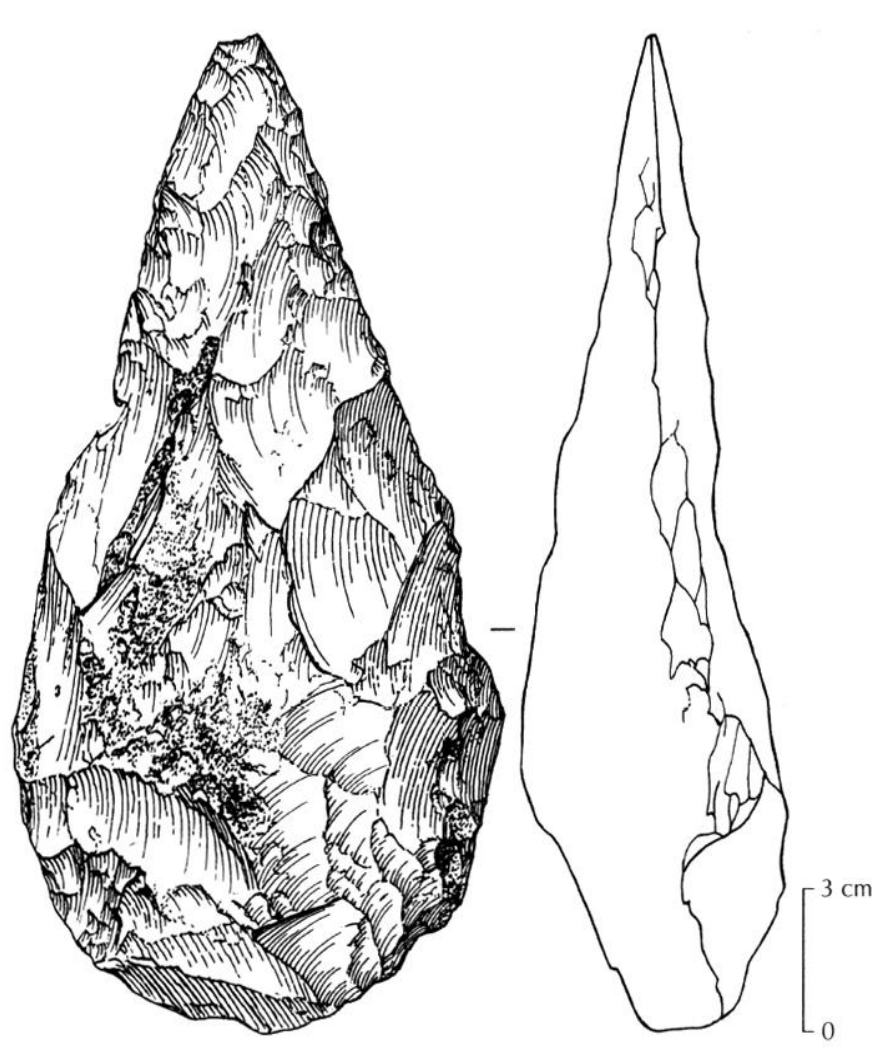

Fig. 3 - Biface acheuleen (Saint-Ferme).

Dordogne et L. Moisan en a fourni une description détaillée (op. cit.). Les séries les plus abondantes ont été attribuées à l'Acheuléen supérieur et ont été datées du Riss III. Elles coexistent parfois dans la masse des cailloutis avec des industries roulées plus anciennes. Ces industries peu Levallois sont relativement riches en bifaces, pour la plupart de facture sommaire, nucléiformes, associés à des outils sur galets dont certains évoquent plutôt des nucléus, outre la présence occasionnelle de hachereaux sur éclats. Au cours des dernières années, les prospections de F. et $\mathrm{D}$. Millet sur les formations alluviales du vignoble des Graves de Bordeaux ont permis de recueillir en surface des séries abondantes issues de la moyenne terrasse mindélienne (Millet, Millet, 1991). Ces industries d'allure archaïque, qui exploitent les galets de quartz et de quartzite de la terrasse, comportent surtout des unifaces, outils ou nucléus, tandis que les bifaces, le plus souvent partiels, sont rares. Le débitage Levallois n'est pas représenté et les outils sur éclats sont pratiquement absents.

Les plateaux de la rive droite de la Dordogne dans la région foyenne et ceux de la moitié orientale de l'Entre-
Deux-Mers, à l'est de la vallée de l'Engranne, livrent en plein air des indices abondants d'Acheuléen (fig. 2 et 3). Les découvertes anciennes concernent le plus souvent des bifaces isolés. Des exemplaires d'excellente facture pourraient appartenir à l'Acheuléen final (fig. 3), voire même au Moustérien de tradition acheuléenne, et apparaissent souvent mélangés en surface à d'autres industries, sur des sites détruits par les labours. En outre, les industries rissiennes, pauvres en bifaces de type acheuléen, peuvent être rattachées au Paléolithique moyen. L'outillage sur éclats est relativement moins bien représenté que les bifaces dans les séries issues de récoltes anciennes, mais probablement a-t-il été en partie négligé par les prospecteurs. Les découvertes récentes qui ont donné lieu à des ramassages exhaustifs confirment cependant la faible diversification de l'outillage commun. Implantés sur des gîtes de matière première locale, silex lacustre en plaquettes de l'Aquitanien, silex du calcaire lacustre de Castillon ou du toit de l'Oligocène, ces gisements correspondent pour la plupart à des ateliers de taille, riches en produits bruts de débitage et en débris, tandis que les outils sont rares et les bifaces absents ou sporadiques, de facture sommaire, nucléiformes, partiels. Le débitage Levallois est largement représenté et il s'agit d'un mode de débitage récurrent centripète sur plaquettes dont les pans naturels latéraux ont fréquemment servi de plans de frappe. Les industries acheuléennes ne sont pour l'instant pas connues sous abri en Gironde et les éléments recueillis dans le remplissage des rivières souterraines y sont en position secondaire et proviennent des limons de couverture des plateaux.

\section{LE MOUSTÉRIEN DU WÜRM ANCIEN}

Les dépôts du Würm ancien qui ont livré des industries moustériennes sont presque exclusivement de plein air à l'exception des couches profondes de la grotte de Pair-non-Pair à Marcamps (Prignac-et-Marcamps) où F. Daleau a rassemblé des séries globales, visiblement mélangées, associant des éléments appartenant au Moustérien de tradition acheuléenne (fig. 4) à du Moustérien Quina (Lenoir, 1983). Les industries du Würm ancien sont mal représentées dans les terrasses alluviales du Libournais (Moisan, 1978a, 1978b) tandis que les découvertes à la surface des plateaux de la rive droite de la Dordogne et de l'Entre-Deux-Mers sont plus 

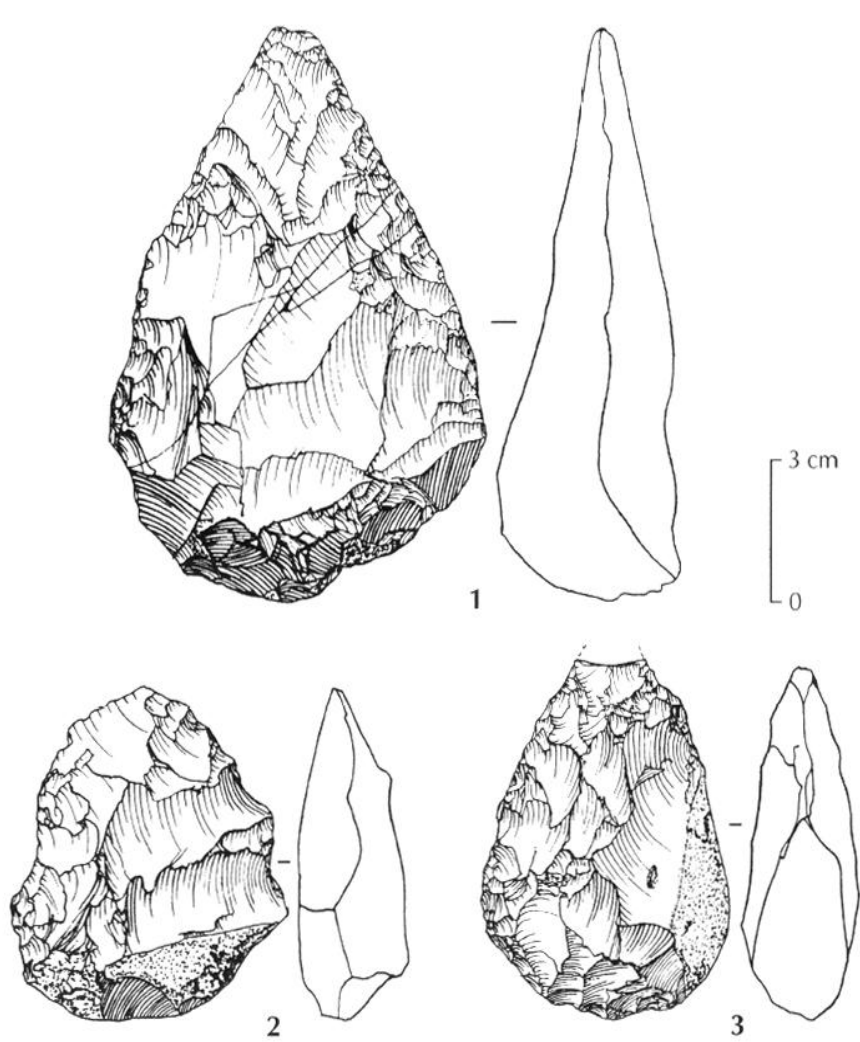

Fig. 4 - Bifaces cordiformes de la grotte de Pair-non-Pair, Marcamps, (musée d'Aquitaine, coll. Daleau) : 1, couche D'; 2, couche KI); 3, couche $K D$ :

nombreuses. Rares cependant sont les ensembles numériquement représentatifs, à l'exception des séries du Moustérien de tradition acheuléenne de Cornemps et de Puynormand (Lenoir, 1983), qui ne présentent pas de parfaites garanties d'homogénéité, et de l'industrie de Chinchon, Castillon-la-Bataille, tout à fait comparable au Moustérien Quina des couches 22 à 24 de Combe-Grenal, Domme (Dordogne) (Sireix, Bordes, 1972). Isolés des autres gisements du Paléolithique moyen de la Gironde, les sites implantés sur les gîtes de silex campanien du bombement anticlinal de Villagrains, Cabanac-etVillagrains, en rive gauche de la Dordogne, au cour de la haute lande girondine, revêtent ainsi un intérêt particulier (Legigan et al., 1978 ; Legigan, Lenoir, 1990 ; Lenoir, 1983, 1985). Les industries moustériennes (fig. 5) y sont pour majeure partie façonnées en silex local, outre une composante plus discrète de silex noir recueilli sous forme de petits galets dans des dépôts alluviaux et de silex lacustre tertiaire parrenu dans le site sous forme de produits Levallois. Ces industries sont relativement paurres en outils par rapport à la masse importante de produits de débitage bruts et de déchets de taille. Le débitage est Levallois récurrent centripète avec tous les intermédiaires entre des nucléus sur éclats ne portant que quelques enlèvements sur la face inférieure de l'éclat et les nucléus centripètes très exploités. L'outillage montre une représentation équilibrée des racloirs et des pièces à encoche et denticulés. Les racloirs, généralement de bonne facture, sont à retouche ordinaire ou légèrement écailleuse, rarement Quina. Ces industries rentrent dans la définition du Moustérien typique proposée par F. Bordes (1953, 1981). Le gisement de Camiac, Camiac-et-Saint-Denis, contemporain de l'interstade würmien (Lenoir, 1980, 1983; Guadelli, 1987; Guadelli et al., 1988 ; Vivent, 1988 ; Lenoir, 1990), a livré une faune abondante et variée associée à un repaire d'hyène. Une industrie lithique peu abondante provient de la même couche que la faune, mais elle ne lui est probablement pas synchrone. Non Levallois, elle se caractérise par la fréquence d'éclats courts débordants, à talon épais, de morphologie identique à celles de pointes pseudo-levallois épaisses. Les nucléus sont épais, globuleux ou informes sans exemplaires nettement discoïdes ou à enlèvements centripètes. L'outillage comporte des racloirs, des pièces à encoche et des denticulés, une pointe à dos, un grattoir, un burin atypique, deux fragments de lames retouchées, deux éclats tronqués et un éclat bitronqué, une raclette, deux outils sur galets. Plusieurs esquilles osseuses présentent un poli à l'extrémité qui est dû à l'ingestion par l'hỵène et qui ne résulte pas d'une action anthropique. Nous disposons d'un résultat de datation effectuée sur os par la méthode classique $\mathrm{du}^{14} \mathrm{C}$ (Ly $1104: 35100 \pm 2000$-1500 BP).

\section{LE CHÂTELPERRONIEN}

Le Paléolithique supérieur le plus ancien (Châtelperronien) demeure relativement discret dans les basses vallées de la Garonne et de la Dordogne (fig. 6). Il était représenté dans la grotte de Pair-non-Pair à Marcamps dans des niveaux d'habitat postérieurs à l'effondrement du plafond du grand couloir, mais le matériel qui en provient est mélangé à celui des niveaux aurignaciens et moustériens. Plusieurs pointes de Châtelperron (fig. 7) ont été recueillies dans les couches profondes du couloir extérieur, plus particulièrement 

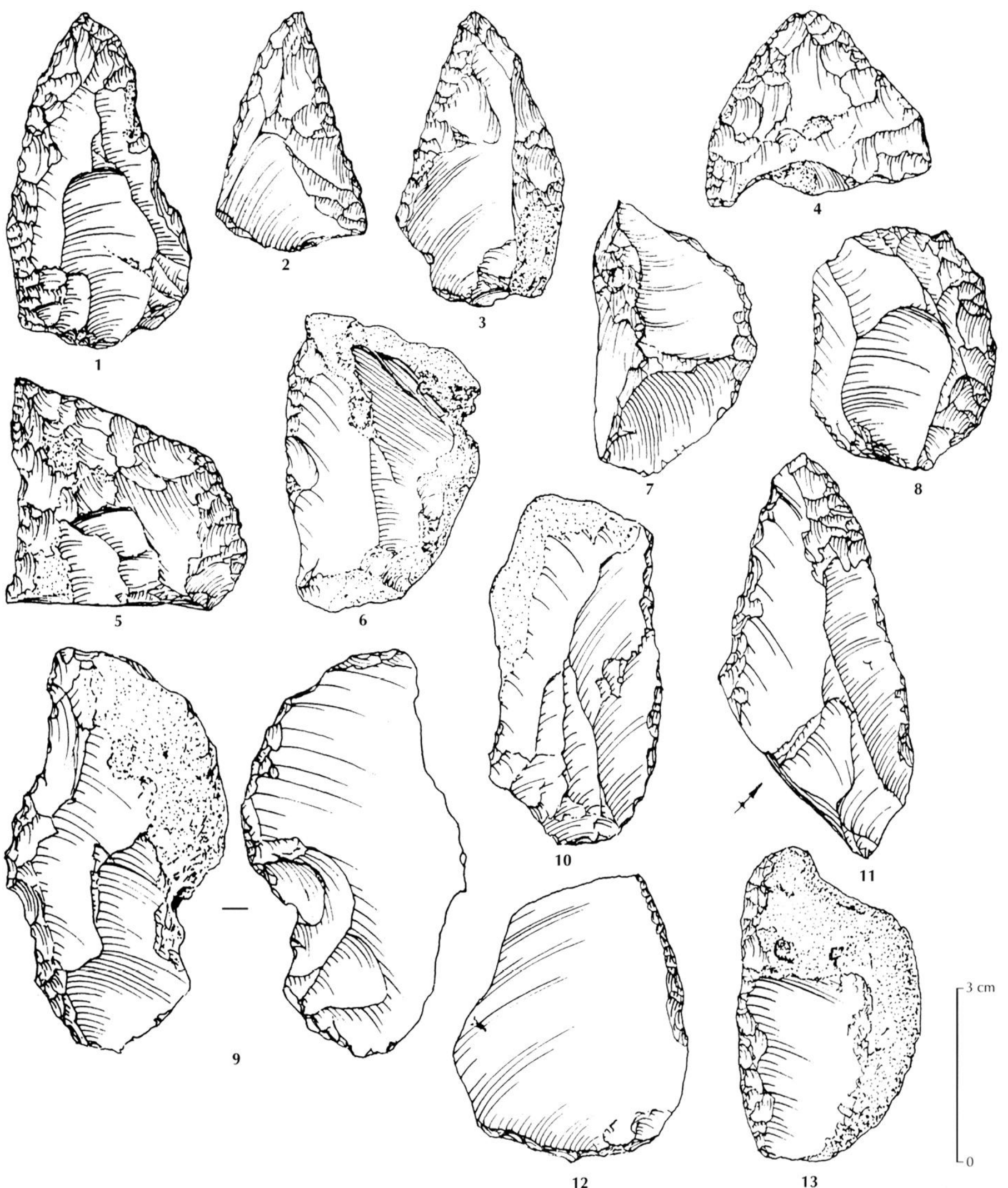

Fig. 5 - Industrie lithique moustérienne de Villagrains: 1, 2, pointes moustérienness ; 3, 4, racloirs convergents ; 5, 11, racloirs déjetés ; $6-8,10,13$, racloirs simples; 9, racloir à dos aminci; 12, racloir sur face plane. 
dans la couche D', tandis que le remplissage de la partie non effondrée en était plus paure (Daleau, 1881; Chernier, 1963 ; Sonneville-Bordes, 1965 ; Lenoir, 1983). Un couteau de Châtelperron provient d'une des couches profondes aurignaciennes (C.7) du talus du Roc de Marcamps près de Pair-non-Pair (Lenoir, 1983). Dans la vallée de l'Euille, affluent de la rive droite de la Garonne, la grotte de Haurets à Iadaux (Breuil, 1911 ; Labrie, 1905, 1923 ; Malvesin-Fabre, 1946 ; Lenoir, 1983, 1986) a livré dans la zone du porche des pointes à dos de type Châtelperron associées à des racloirs ainsi qu'à un fragment de lame étranglée (Breuil, 1911), tandis que les galeries plus profondes ont servi de repaire d'hyène comme l'ont confirmé nos propres recherches dans ce gisement. D'autres cavités appartenant au bassin versant de la basse vallée de la Garonne (La Mothe à Cénac, Lavison à Saint-Maixant) paraissent également avoir été fréquentées par l'hỵène et peut-être occasionnellement par l'homme.

\section{AURIGNACIENS ET GRAVETTIENS}

L'Aurignacien demeure pour l'instant mal caractérisé en Gironde car les séries de la grotte de Pair-non-Pair ne sont pas homogènes et elles ont fait l'objet d'un tri après la mort de Daleau. Connu dans plusieurs gisements de plein air (fig. 6), remaniés par les labours (Lenoir, 1983), il était représenté dans deux petits abris de l'Entre-DeuxMers, situés de part et d'autre d'un petit affluent de la Durèze près de son débouché sur la plaine alluviale de la Dordogne. Le premier abri fouillé anciennement (Brial et al., 1954) a livré une molaire humaine (Gambier, Lenoir, 1991) dans un contexte de faune abondante, à Cheval, Renne, Rhinocéros, associée à une industrie lithique et osseuse typiquement aurignacienne outre des indices gravettiens (fléchette), l'autre abri a fait l'objet d'une fouille de sauvetage récente (Turq, Lenoir, 1992). À proximité immédiate de Pair-non-Pair, les couches profondes du talus du Roc de Marcamps ont livré une industrie aurignacienne trop peu abondante pour permettre une caractérisation précise mais qui a pu être datée (Ly $2682: 26500 \pm 1400 \mathrm{BP}$ pour la couche 8). La grotte de Jolias (Daleau, Gassies, 1874) toute proche, aujourd'hui détruite, a livré de l'industrie (fig. 8) et de la faune. L'industrie conservée au Musée d'Aquitaine provient de plusieurs points de récolte et n'est probablement pas

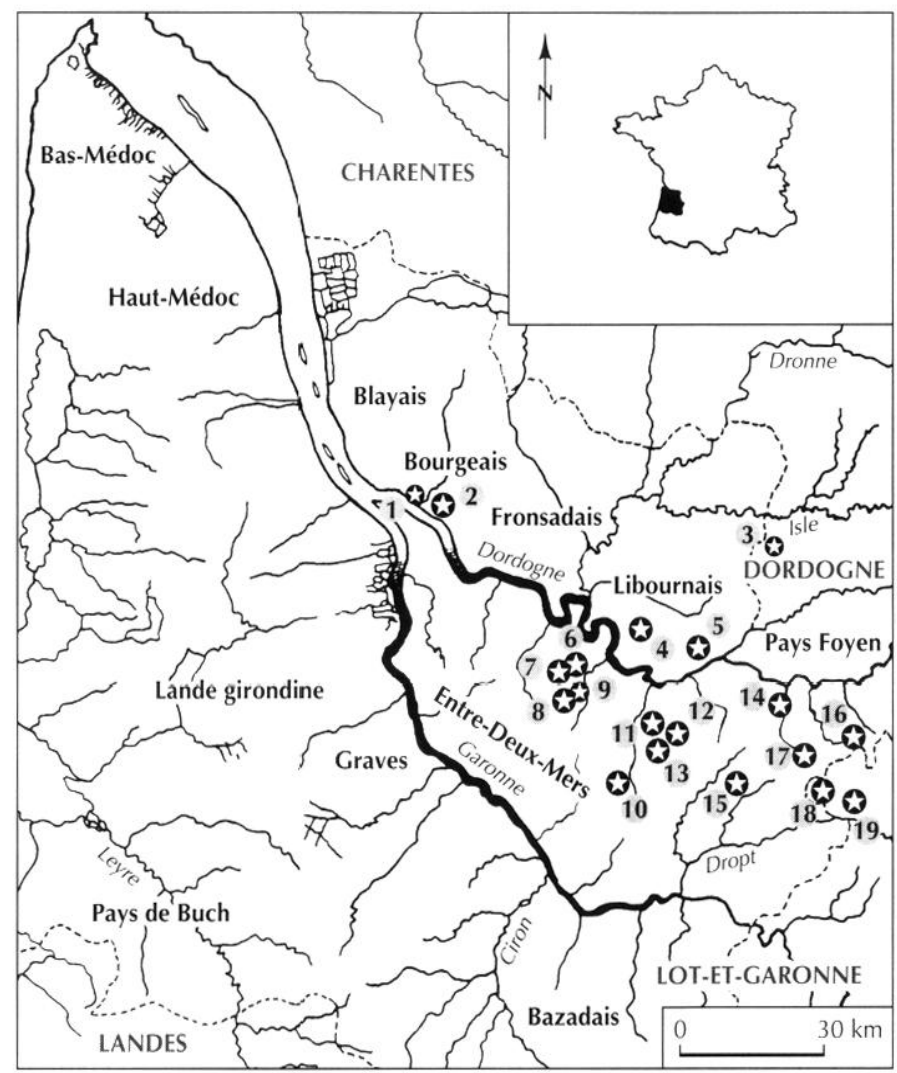

Fig. 6 - Principaux gisements du Paléolithique supérieur ancien des basses vallées de la Dordogne et de la Garonne (Au : Aurignacien, (G: Gravettion, S : Solutréen) : 1, La Lustre (G) ; 2, Pair-non-Pair (G et $A u)$, Le Roc de Marcamps (Au) et Jolias $(A u) ; 3$, La Bernarderie $(G) ; 4$, Fonplégade $(A u)$; 5 , Camp de la Hire $(G)$; 6, abri Lespaux (G); 7 , plateau de Larroque (Au);8, Les Artigaux (G) ; 9, Camiac (Au, (.) ; 10, Fransois Brugier (G) ; 11, Le GrandMoulin (S) ; 12, Pontaret (G), 13, Milha (G) ; 14, Durege et le Pigeonnier $(A u) ; 15$, Launay $(G) ; 16$, Vignes du Moulin $(A u, G)$; 17, Laforêt (Au); 18, Crand-Champ $(A u) ; 19$, Jacob (C).

homogène. Elle se caractérise cependant par une nette composante aurignacienne avec des grattoirs épais, des lames à retouche aurignacienne, parfois à étranglement (Lenoir, 1983).

Le Gravettien est connu dans des gisements de plein air et sous abri (fig. 6), outre les niveaux de la grotte de Pair-non-Pair, mais les séries qui lui appartiennent sont pour la plupart mélangées à d'autres industries, comme à Pair-non-Pair, ou proviennent de récoltes de surface sur des sites remaniés par les labours. Deux gisements du bassin de la Canodonne dans l'Entre-Deux-Mers ont cependant fait l'objet de fouilles : le gisement de plein air des Artigaux à Camiac-et-Saint-Denis (Lenoir, 1977, 1983) qui a livré du Périgordien à burins de Bassaler et l'abri 

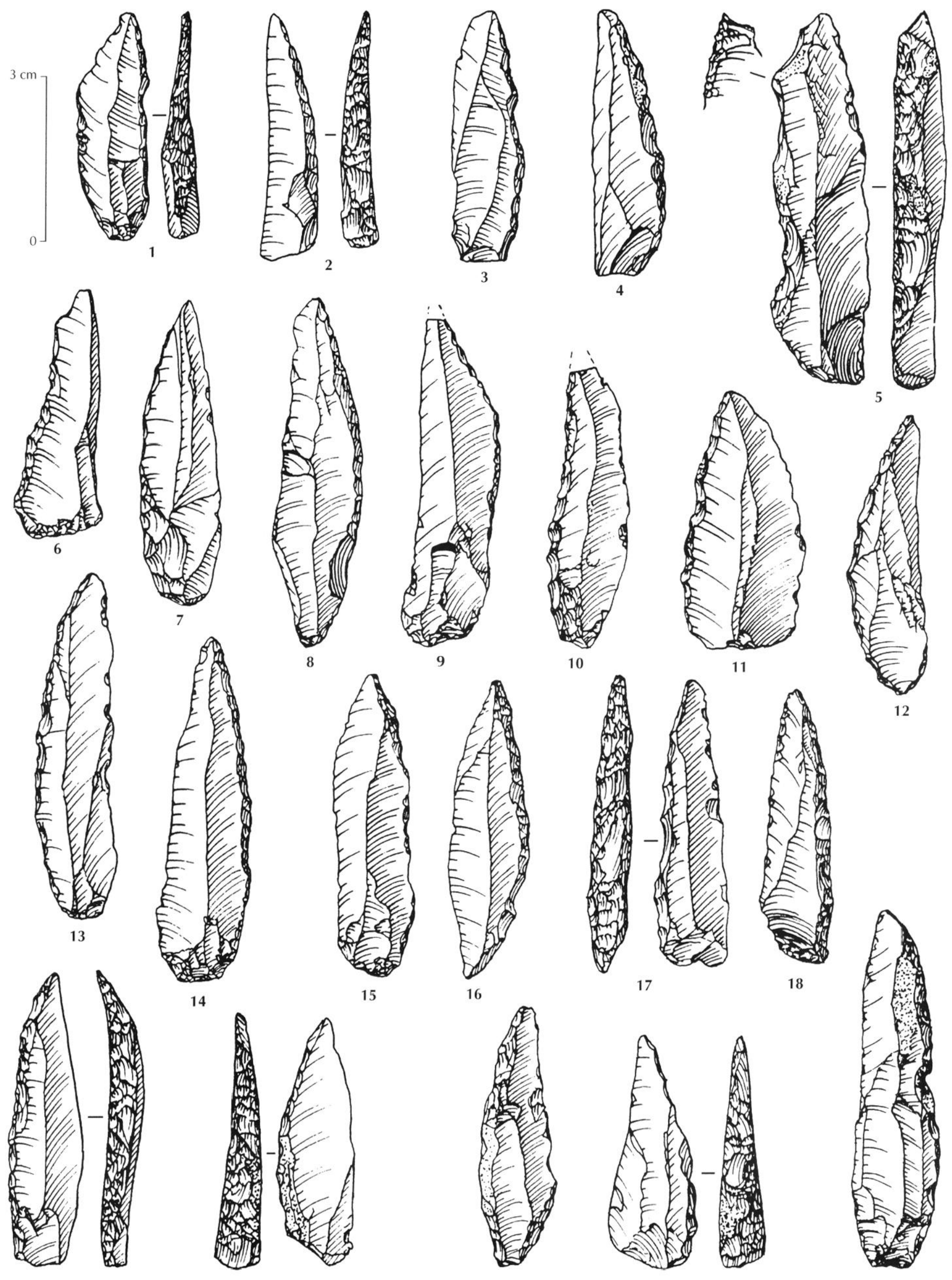

20

22

23

Fig. 7 - (srolte de Pair-non-Pair à Marcamps : pointes de Châtelperron de Lu couche I)' (musée d'Aquitaine, coll. Daleau). 

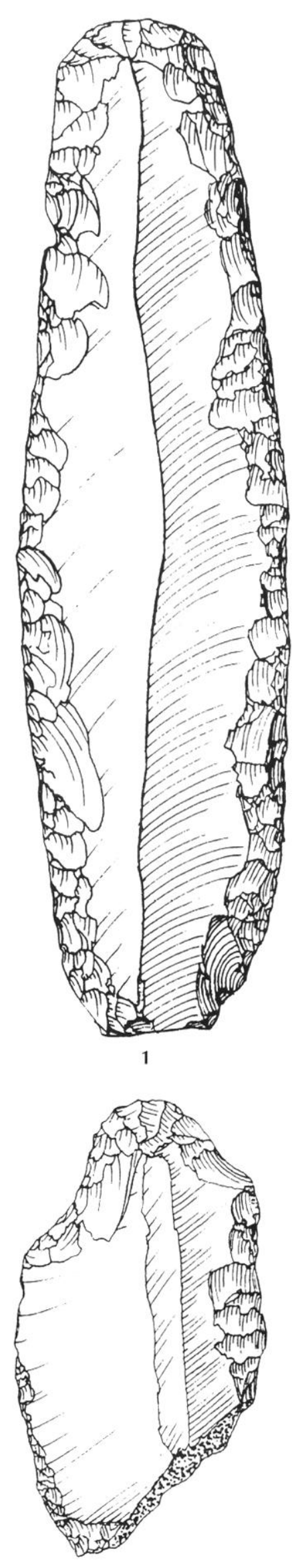

6

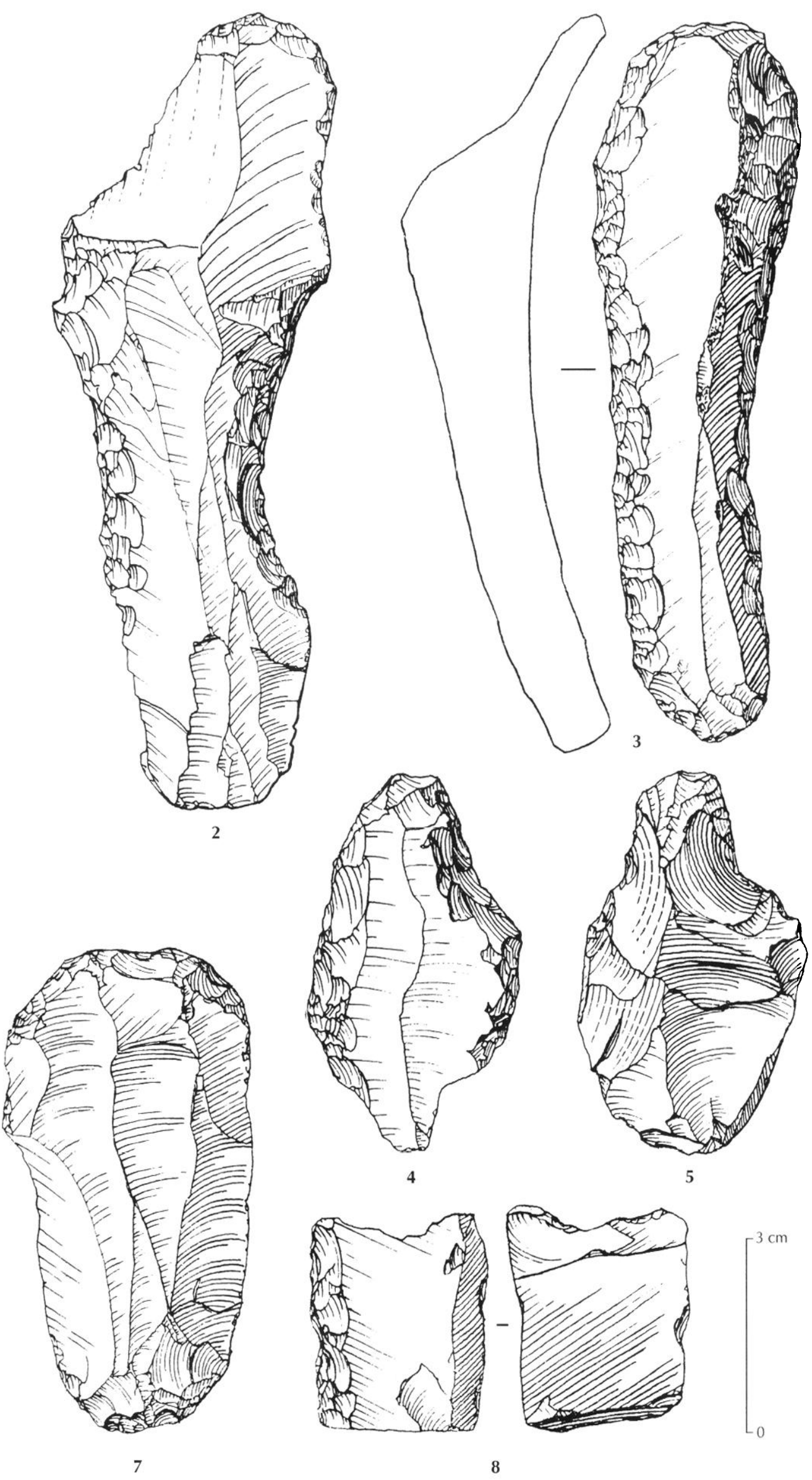

Fig. 8 - Industrie lithique de la grotle de Jolias à Marcamps, (musée dAquitaine, coll. Daténu), : 1-3, grattoirs sur lame aurignacienne; 4-6, gralloirs à museau; T, gratloir double ; 8, lame tromquée et retouchét. 
Lespaux à Saint-Quentin-de-Baron (Cousté, Krtolitza, 1965 ; Krtolitza, Lenoir, 1998).

\section{L'ABRI LESPAUX}

Découvert en 1943 par R. Cousté dans la commune de Saint-Quentin-de-Baron, il se situe en rive droite du ruisseau de l'Esteil, petit affluent de la Canodonne qui rejoint la plaine alluviale de la basse vallée de la Dordogne au droit de Moulon. C'est un petit abri sous roche creusé dans le calcaire à Astéries (Stampien) coiffant un banc d'argile. Cette cavité large de $8 \mathrm{~m}$ en façade sur $9 \mathrm{~m}$ de profondeur s'ouvre à l'ouest-sud-ouest. Son plancher forme une terrasse qui domine de $4,50 \mathrm{~m}$ environ le fond actuel du vallon. Le remplissage reposait sur cette terrasse et se prolongeait vers le fond de l'abri sur une longueur d'environ 8 mètres. Les fouilles effectuées de 1960 à 1966 par Y. Krtolitza ont concerné plusieurs niveaux de Périgordien à burins de Noailles. La faune comporte des restes de Cheval, Renne, grands bovidés. La séquence stratigraphique observée lors de ces travaux est la suivante de haut en bas (Cousté, Krtolitza, 1965; Y. Krtolitza, documents de fouille inédits) :

- couche 6 , terre végétale (ép. : $60 \mathrm{~cm}$ ) avec os et silex remaniés, débris de poterie de l'Âge du Fer, traces de gallo-romain ;

- couche 5, menus éboulis avec quelques blocs, assez rares silex ;

- couche 4, couche de couleur brun clair d'aspect sableux, qui se superposait directement à la couche sousjacente (épaisseur variant entre 10 et $30 \mathrm{~cm}$ ) ;

- couche 3, couche ocre-rouge foncé, d'aspect sableux dans la partie supérieure et bréchiforme à la base ; cette couche, dont l'épaisseur maximum atteignait $40 \mathrm{~cm}$, se terminait en biseau en bordure de la terrasse ; elle renfermait à sa base un foyer long de $3 \mathrm{~m}$ et épais de $10 \mathrm{~cm}$. Ce foyer apparaissait à $2 \mathrm{~m}$ en retrait de l'escarpement rocheux et recouvrait directement à cet endroit le plancher calcaire, tandis qu'il reposait sur la couche 2 en fond d'abri ;

- couche 2, couche gris-jaune formant une lentille de $2 \mathrm{~m}$ de long, $1,50 \mathrm{~m}$ de large et $25 \mathrm{~cm}$ d'épaisseur au centre du gisement et une petite lentille piégée dans un creux du plancher rocheux en fond d'abri ;

- couche 1, couche jaunâtre stérile, d'aspect marneux, formée par le délitage du sol rocheux.
Toutes les couches, excepté la couche 1, ont livré une industrie du Périgordien supérieur à burins de Noailles mêlée à des vestiges modernes dans la couche 6 .

Outre quelques mentions sporadiques (David, 1966 ; Lenoir, 1983) et une courte étude préliminaire de R. Cousté et Y. Krtolitza qui concerne une partie seulement des vestiges recueillis, ce gisement a fait l'objet d'autres campagnes de fouille mais demeurait inédit. Ainsi le matériel archéologique qui en provient apparaissait d'un intérêt particulier compte tenu de la rareté des gisements gravettiens en Gironde et nous en avons donc récemment repris l'étude arec le fouilleur. L'industrie de la couche 5 est peu abondante ( 15 outils seulement), celle de la couche 4 comporte 164 outils dont une cinquantaine de burins de Noailles et une trentaine de pointes à dos. La couche 3 prise dans son ensemble comporte 2170 outils retouchés dont un grand nombre de pointes à dos (317) et de burins de Noailles (440), le tout associé à une quantité importante de produits de débitage et de déchets. La couche de base (couche 2), plus pauvre (250) outils), se singularise par la présence de fléchettes et d'une pointe de Font-Robert.

Dans tous ces niveaux, l'industrie lithique, en majeure partie façonnée sur des silex recueillis sous forme de galets dans les alluvions de la Dordogne, est laminaire et lamellaire et elle comporte de nombreux nucléus prismatiques. L'outillage, riche en burins, comporte de nombreux burins de Noailles de types variés parmi lesquels dominent les exemplaires simples sur troncature suivis par les burins de Noailles doubles sur troncature, puis par les burins de Noailles sur cassure pour la plupart simples, les burins triples, un seul quadruple et quelques exemplaires mixtes. Il y a des burins de Bassaler. I.es pointes à dos (gravettes et microgravettes) associées dans ces niveaux à des lamelles à dos plus ou moins épais sont en majorité fragmentaires arec une prédominance des parties proximales. Elles portent pour la plupart des retouches d'amincissement sur la face inférieure à l'extrémité distale et dans la partie proximale qui partent des deux bords ou du bord opposé au dos, plus rarement du dos. Le dos est à retouche abrupte ou semi-abrupte directe ou croisée le plus souvent dextre. Le bord opposé porte fréquemment une retouche directe abrupte ou semi-abrupte ou une fine retouche marginale. L'industrie osseuse comporte notamment une flûte en os associée à des lissoirs, des sagaies (dont un exemplaire de type Isturitz), des poinçons en os, des alènes, des 
baguettes, des côtes de renne sciées et polies portant des séries de petits traits gravés, des bâtons percés, ainsi que des éléments de parures : canines de renard et canines de félin percées, coquilles de Neritina percées, craches de cerf percées à la racine dont deux exemplaires sont des imitations en os (couche 2).

Dans l'ensemble, l'industrie lithique et osseuse de l'abri Lespaux montre des traits communs avec celle des niveaux gravettiens d'Isturitz. (Pyrénées-Atlantiques). Il y a des burins de Noailles, des gravettes et des microgravettes dans tous les niveaux, des burins de Bassaler dans les couches 3 et 4 , une pointe de Font-Robert et quatre fléchettes dans la couche 2. L'industrie lithique ressemble à celle d'autres gisements gravettiens à burins de Noailles et montre des ressemblances arec celle du niveau 4 de l'abri Pataud, Les-Eyzies-de-Tavac (Dordogne) (Darid, 1985), notamment par l'abondance des burins de Noailles et la présence de burins de Bassaler (burins du Raysse) mais elle en diffère cependant par l'absence des burins à modification tertiaire et des burins-pointes (David, 1966, 1985; Bricker éd., 1995). En revanche, l'industrie osseuse montre des caractères communs pour l'abri Lespaux, l'abri Pataud et la grotte d'Isturitz (Passemard, 1944), notamment en ce qui concerne la présence de sagaies d'Isturitz dans le site éponyme et dans les deux autres gisements, et celle de diaphyses sciées et polies qui portent des entailles plus ou moins profondes anciennement interprétées comme des marques de chasse pour les couches gravettiennes de Pair-non-Pair : couche 3 , couche 3 supérieure, couche 7 (Cheynier, 1963, fig. 14, 19, 59, 60) et qui sont également représentées à Isturitz (Passemard, 1944, pl. XI, XIII ; Saint-Périer, Saint-Périer, 1952 ; Esparza San Juan, 1995, fig. 45-47). Ce dernier gisement, tout comme Lespaux et Pair-non-Pair, a livré une flûte en os (Passemard, 1944, pl. VII ; Cheynier, 1963, fig. 22 ; Roussot, 1970).

\section{L'ÉPISODE SOLUTRÉEN}

Le Solutréen est assez. mal connu dans les basses vallées de la Dordogne (fig. 6), si ce n'est par quelques indices sporadiques ou mêlés en surface à d'autres industries du Paléolithique supérieur (Lenoir, 1974, 1983; Blanchard et al., 1976). Cependant, sa présence, quelque temps contestée dans le talus du Grand-Moulin à Lugasson (Smith, 1966), y a été confirmée par nos recherches (Lenoir, 1982, 1983). De ce gisement, que les fouilles anciennes de J. Labrie semblent avoir épuisé, proviennent plusieurs grandes pièces foliacées bifaces (Ferrier, 1938; Lenoir, 1982, 1983) et le Solutréen y précède les Badegouliens et les Magdaléniens.

\section{BADEGOULIENS ET MAGDALÉNIENS}

C'est pour la fin du Paléolithique que nous disposons des données les plus complètes et les plus significatives, tant en ce qui concerne l'occupation des grottes et des abris sous roche que les activités de subsistance, l'art mobilier, les territoires.

Dans les bassins inférieurs de la Dordogne et de la Garonne, les gisements badegouliens sont pour la plupart de petits gisements de plein air, sur des points hauts, des buttes témoins de calcaires lacustres aquitaniens, de hautes terrasses alluviales, ils sont plus rarement placés en pied de falaise (abri Houleau à Sainte-Florence, Le Grand-Moulin à Lugasson), ou en bas de pente (un seul cas, à proximité immédiate d'une source). Tout comme pour le Magdalénien, l'occupation est nettement plus dense sur le bassin-versant de la Dordogne que sur celui de la Garonne et elle apparaît très sporadique entre Garonne et océan (fig. 9).

Les gisements de plein air sont connus par des ensembles lithiques d'importance inégale (Lenoir, 1983, 1988) recueillis en surface sur des sites remaniés par les labours et qui ne présentent donc pas toujours de parfaites garanties d'homogénéité, avec notamment pour quelques cas des présomptions de mélanges avec du Gravettien. Dans l'ensemble, ce sont des industries (fig. 10) peu lamellaires, peu laminaires, avec des lames courtes, des outils sur éclats, parfois de nombreux petits éclats plats et minces qui ont servi de pièces-supports aux raclettes (Birac, Pourquey et Gregeons à Castelviel). Généralement, les burins sont plus nombreux que les grattoirs excepté dans deux sites. Ces burins sont pour la plupart dièdres ou sur cassure, parfois multiples, plus rarement sur troncature retouchée avec des burins transversaux parfois abondants. Parmi les grattoirs, certains exemplaires épais, rappelant des grattoirs aurignaciens, pourraient être des nucléus à lamelles. Il $y$ a des racloirs d'allure moustérienne (Beauregard à Mazères), des pièces à encoche et des denticulés, des lames retouchées, parfois à retouche écailleuse, des lames tronquées le plus 


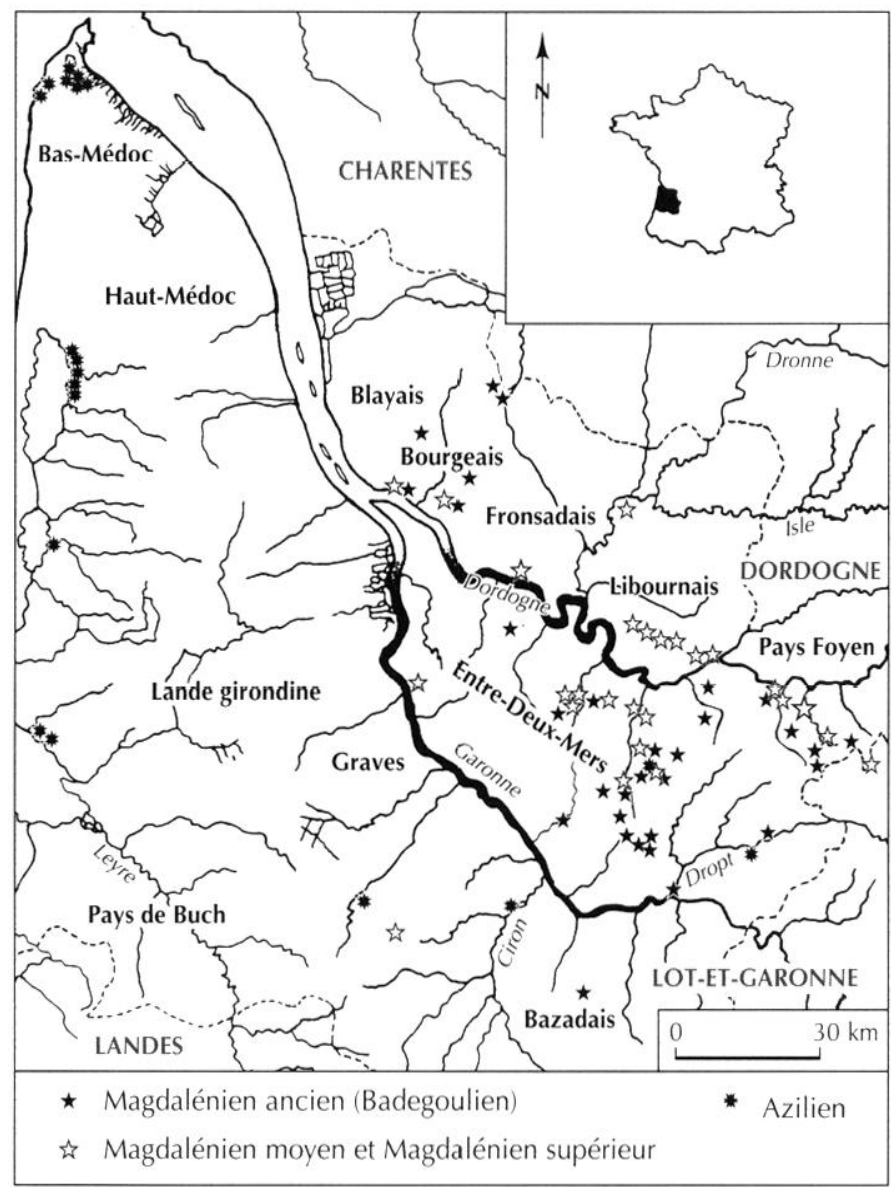

Fig. 9 - Répartition des gisements du Badegoulien, du Magdalénien el de l'Azilien dans les basses vallées de la Dordogne et de la Garonne.

souvent à troncature retouchée partielle. Les perçoirs et les becs sont peu nombreux, parfois multiples, mais rarement en étoile. Les outils composites sont surtout représentés par des grattoirs-burins. Les pièces esquillées sont parfois abondantes (La Bertonne à Peujard). Les raclettes sont nombreuses dans certains sites (Houleau, Birac, Pourquey). Des pièces d'un type particulier, les pièces de La Bertonne (Lenoir, 1976, 1983), sont très abondantes dans le site éponyme (Daleau, 1910) et connues plus sporadiquement dans d'autres gisements girondins. Les ensembles lithiques badegouliens de ce secteur apparaissent ainsi très polymorphes et il est difficile de leur attribuer une position chronologique précise en l'absence de restes osseux qui ne se sont pas conservés dans les gisements de plein air. Parmi tous ces gisements, celui de Beauregard à Mazères apparaît quelque peu marginal, tant par sa position géographique (à l'ouest de la Garonne) que par l'abondance des racloirs qui sem-
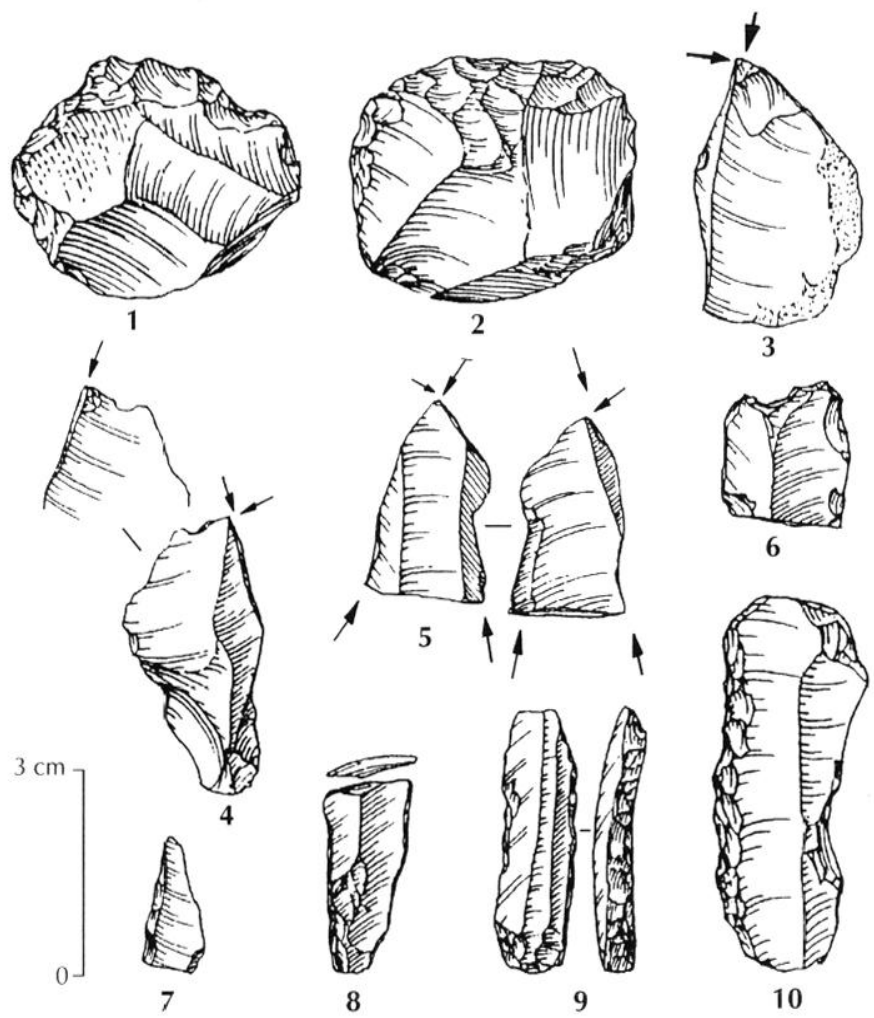

6

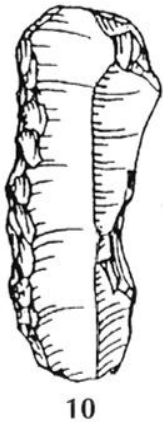

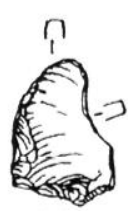

11

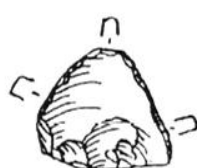

12

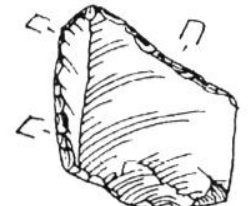

13

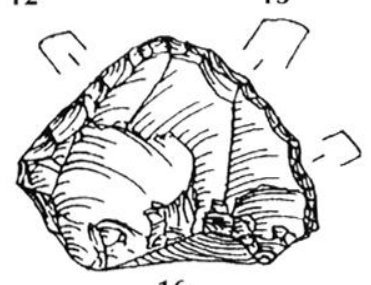

16

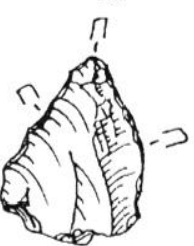

14

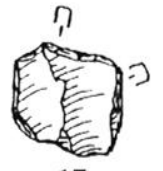

17
Fig. 10 - Industrie lithique badegoulieme de Balette à Bellebat : 1. 2, grattoirs sur éclat ; 3-5, burins dièdres; 6, extrémité de lame tronqué, 7, fragment de petile pièce ì dos; 10, lame retouchie ; 8, 9, 11-17 raclettes.

blent cependant bien constituer une composante de cette industrie. C'est dans ce gisement qu'outre le silex lacustre aquitanien local ont été exploités des silex sénoniens de provenance éloignée qui pourraient provenir des anticlinaux de Chalosse (Lenoir et al., 1997).

Si les petits habitats du Badegoulien sont presque exclusivement placés sur des points hauts, ceux du Magdalénien ancien et moyen sont souvent sous abri ou 


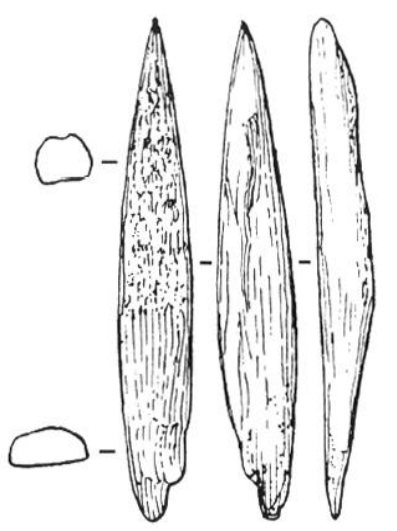

1

Fig. 11 - Industrie osseuse de la grotte des Fíes à Marramps, (musée didquitaine, coll. I)aleau): 1-6, 8, sagaies; 9, aiguille à chas; 7 , plaquetle en os polie et graviée.
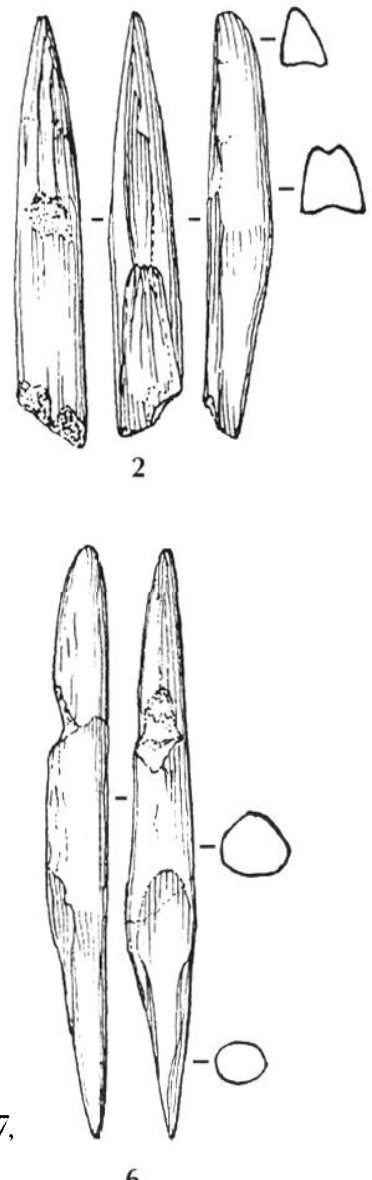

6

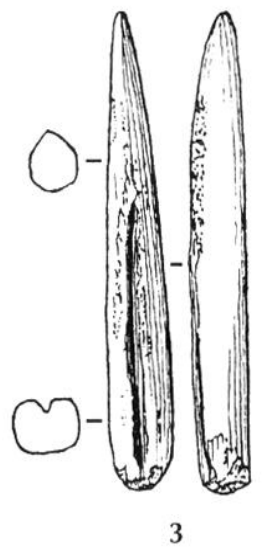

3

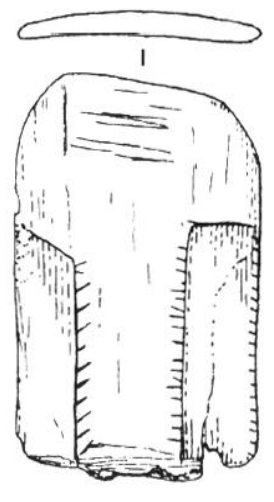

7
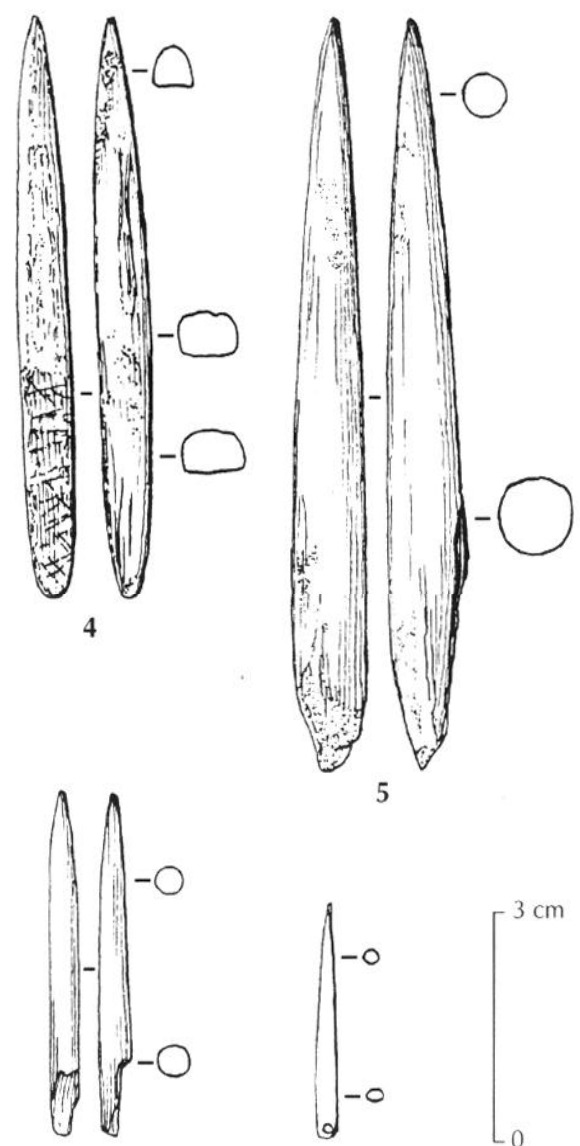

8

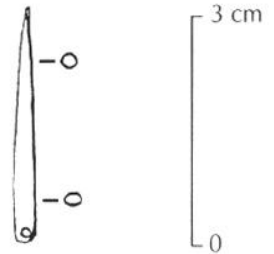

9 en pied de falaise (fig. 9). Pendant le Dryas ancien, alors que sur les coteaux de Gironde régnaient des conditions steppiques, froides et sèches, propices à la présence de grands troupeaux d'antilope saiga, l'occupation humaine s'intensifie comme en témoignent les grands sites de la rive droite de la Dordogne tels que Le Roc de Marcamps et Saint-Germain-la-Rivière, et les gisements plus modestes sous abris dans les vallons de l'Entre-DeuxMers. Les gisements magdaléniens de plein air connus sur les versants bien exposés des coteaux de la rive droite de la Dordogne demeurent quant à eux mal datés en l'absence de matériel osseux, mais l'industrie lithique qu'ils ont livrée rappelle cependant pour la plupart d'entre eux le Magdalénien supérieur.

Les gisements du Magdalénien ancien et moyen en grotte, sous abri ou en pied de falaise, dont la plupart ont fait l'objet de fouilles anciennes (L.e Roc de Marcamps, Saint-Germain-la-Rivière, Jaurias et Moulin-Neuf à SaintQuentin-de-Baron, abri Houleau), ont donné lieu au cours des dernières années à des révisions qui ont permis de mieux définir la nature des industries et des faunes qui y sont conservées, d'établir leur position chronologique et de montrer leurs potentialités pour des recherches futures.

\section{LE ROC DE MARCAMPS}

Découvert en 1929 par P. David et G. Malvesin-rabre, le gisement du Roc de Marcamps à Prignac-et-Marcamps se situe dans le talus en contrebas de la grotte des Fées fouillée par F. Daleau en 1874 (Daleau, 1874). Des fouilles y furent entreprises par les membres de l'école de fouilles de la Société linnéenne de Bordeaux, puis par A. Nicolaï, J. Ferrier de 1933 à 1940, E. Guichard, G. Maziaud jusqu'en 1942, P. Forestier (Roussot, Ferrier, 1970), puis le site fut abandonné jusqu'à ce que nous : reprenions des recherches en 1978. Longtemps considéré comme le talus de la grotte des Fées (Lacorre, 
1938 ; Cousté, 1959a, 1959b ; Roussot, Ferrier, 1970), Le Roc de Marcamps en est en réalité indépendant ainsi que l'ont confirmé nos travaux (Lenoir, 1983, 1993a, 1993b).

Vidée de son remplissage lors des fouilles anciennes, la grotte des Fées renfermait probablement une séquence de Magdalénien moyen et supérieur. La série qui en provient (musée d'Aquitaine) est malheureusement globale (fig. 11) et ne porte pas l'indication des niveaux distingués par $\mathrm{F}$. Daleau lors de la fouille. Lui appartiennent notamment deux fragments de harpons, des sagaies courtes à rainure, des pointes aziliennes, des serpettes de type Loubressac (Lwoff, 1962).

Les anciens fouilleurs n'avaient donné qu'un nombre restreint d'informations sur la stratigraphie des dépôts du Roc de Marcamps et sur la nature des vestiges associés. Les travaux récents ont en grande partie comblé ces lacunes tout en révélant la complexité du gisement. Les fouilles ont été effectuées au Roc de Marcamps de 1979 à 1988 et elles ont concerné deux secteurs séparés d'une trentaine de mètres.

\section{Premier secteur}

La stratigraphie du premier secteur placé directement en contrebas de la grotte des Fées est la suivante, de haut en bas, sous des apports récents d'épaisseur variable.

\section{Ensemble 1}

Cla : lit de pierraille meuble dans une matrice limonoargileuse qui renferme des vestiges gallo-romains et des vestiges plus récents (ép. : 20-30 cm).

Clb : limon brun foncé à granules et petits éboulis arrondis; mélange de vestiges paléolithiques et plus récents (ép. maximum : $40 \mathrm{~cm}$ ).

C1c: limon sableux riche en granules calcaires et en débris de polypiers. Les vestiges paléolithiques y sont rares, issus de l'érosion des couches sous-jacentes (ép. : $30 \mathrm{~cm}$ ).

D'après les résultats d'études palynologiques (Lenoir, Paquereau, 1986), cet ensemble pourrait être contemporain des débuts du Postglaciaire et de la fin du Dryas récent.

\section{Ensemble 2}

C2a : limon argileux brun-jaune, pauvre en éboulis et en vestiges (ép. : $45-50 \mathrm{~cm}$ ) ; industrie magdalénienne.
L'analyse pollinique a révélé dans ce niveau une augmentation du taux de boisement par rapport à la couche immédiatement sous-jacente. Cela pourrait se rapporter au Bölling.

C2b : limon argileux brun-jaune foncé, riche en petits éboulis et granules calcaires et en petits charbons d'os. Cette couche, concrétionnée par endroits, est riche en industrie et en os et elle témoigne d'après la faune et les pollens de conditions steppiques qui pourraient être celles du Dryas ancien (ép. 20-30 cm). Les dates obtenues sont les suivantes : Ly $2290=14200 \pm 190 \mathrm{BP}$; Ly $2291=14910 \pm 240 \mathrm{BP}$.

C3 : limon argileux brun-jaune plus ou moins foncé, bréchifié par endroits; faune et industrie magdalénienne.

C4: éboulis épais constitué de gros blocs plus ou moins volumineux, dans une matrice limono-argileuse, localement plus sableuse, concrétionnée contre les blocs. Cet éboulis, subdivisé en quatre niveaux (C4a, C4b, C4c, (C4d), renferme dans sa partie médiane un niveau riche en ossements de grands bovidés (C4c). Sa base est plus argileuse, sans rupture nette avec la couche sous-jacente. Trois niveaux ont été datés :

$$
\begin{aligned}
& \text { C4a : Ly } 4219=16840 \pm 520 \mathrm{BP} \\
& \text { C4c : Ly } 2292=17410 \pm 310 \mathrm{BP} \\
& \text { C4d : Ly } 4220=17880 \pm 290 \mathrm{BP} .
\end{aligned}
$$

Les analyses polliniques ont révélé pour $\mathrm{C} 4 \mathrm{a}, \mathrm{C} 4 \mathrm{~b}$, C4d, des conditions steppiques tandis que C4c plus boisé témoigne d'un épisode plus doux.

C.5: éboulis hétérométrique dans une matrice argileuse pauvre en vestiges (épaisseur variable) à industrie magdalénienne, datée (Ly 4221 : $18290 \pm 330$ BP). Les flores de cette couche indiquent une forte humidité et un adoucissement des conditions climatiques par rapport aux couches profondes.

\section{Ensemble inférieur}

Les couches profondes étaient plus difficiles à individualiser en raison de la proximité de la nappe phréatique.

C6 : éboulis localement très meuble à éléments calcaires arrondis et surface corrodée à enduit ferrugineux brun foncé. Les vestiges archéologiques sont rares mais la microfaune est relativement abondante. Présence de blocs parfois redressés.

C7 : limon argileux, très caillouteux, à éboulis arrondis à surface corrodée dans une matrice argilo-sableuse 
brun olive, grisâtre par endroits. L'industrie, aurignacienne, et la faune y sont peu abondantes.

(8: éboulis constitué d'éléments peu rolumineux dans l'ensemble, très émoussés, asse\% rapprochés dans une matrice argileuse brun olive. L'industric aurignacienne y est associée à une faune peu abondante, pauvre en restes déterminables. Cet éboulis daté repose sur l'argile stampienne du substratum. I.es flores des couches 6 , 7,8 sont toutes très déboisées et cet ensemble inférieur témoigne d'un épisode steppique très rigoureux, plus accentué en profondeur.

\section{Les industries lithiques magdaléniennes}

D’importance numérique inégale selon les niveaux, les industries lithiques magdaléniennes provenant des fouilles récentes présentent des caractéristiques communes. Elles ont été façonnées à partir de petits galets de silex sénonien recueillis dans les alluvions de la Dordogne, exploités sous forme de nucléus prismatiques à un plan de frappe ou à deux plans de frappe opposés et qui ont fourni des lames et des lamelles. Les produits et sous-produits du débitage laminaire sont représentés dans le gisement, ce qui indique que les différentes phases de la chaine opératoire y ont été accomplies. L'outillage commun est préférentiellement fait sur lames, mais les outils sur éclats sont cependant relativement nombreux : grattoirs et burins épais, pièces à encoche, denticulés. Très abondantes, les lamelles à dos sont pour la plupart fragmentaires et à dos épais, parfois tronquées ou retouchées sur le bord opposé au dos, rarement scalènes ou denticulées. Les burins, qui constituent le groupe typologique dominant au sein de l'outillage commun, sont surtout des burins dièdres, pour la plupart d'axe ou sur cassure, parfois multiples. Ils sont associés à des grattoirs simples sur lames brutes ou retouchées, tandis que les grattoirs plats sur éclats et les petits grattoirs de type azilien sont rares. Les grattoirs épais de type aurignacien sont représentés en faible nombre dans tous les niveaux, mais il peut s'agir dans de nombreux cas de nucléus à lamelles. Les outils composites sont surtout des grattoirsburins. Les perçoirs et les becs sont rares, de même que les lames à dos. Les lames tronquées sont pour la plupart à troncature retouchée partielle et les lames retouchées sont le plus souvent fragmentaires. I es pièces à encoche et les denticulés, parfois sur éclats épais, complètent l'ensemble tandis que les racloirs et les pièces esquillées sont rares; les raclettes sont sporadiques et atypiques.

\section{L'industrie osseuse}

L industrie osseuse recucillic lors des fouilles récentes est peu abondante et le plus souvent constituée d'outils fragmentaires. Dépourvue de harpons, elle comporte des sagaies à section ovalaire ou quadrangulaire, à biseau simple ou double, strié et à fût rainuré associées à des aiguilles, des poinçons, des baguettes et des fragments de bâtons percés. Nous n'avons pas découvert de navettes dans le secteur concerné par nos travaux.

\section{La faune}

La grande faune (Slott-Moller, 1988) fait clairement apparaître deux sous-ensembles dans la séquence magdalénienne. L'ensemble inférieur (niveaux C.5, C4c, (4d) montre la nette prédominance du groupe de milieu ouvert non arctique sur les autres groupes, avec dominance du Bison au sein d'un ensemble faunique qui témoigne de conditions climatiques pas trop rigoureuses et d'un certain taux d'humidité, surtout en début et en fin de séquence.

L'ensemble supérieur (niveaux C2, C2b, C3, C4a, C4b) se caractérise par la prédominance du groupe de milieu ouvert non arctique. Il s'agit d'une faune steppique dominée par l'Antilope saïga associée au Renne, au Bison, au Cheval, au Spermophile, au Chamois et au Rhinocéros laineux sporadiques. Microlus gregalis (Marquet, 1984) est très abondant dans toute la séquence, ce qui témoigne de conditions climatiques froides et sèches également attestées par la présence du Spermophile. Des espèces forestières apparaissent dans la couche 4 et surtout dans la couche 2 , où le pourcentage de Microtus gregalis diminue un peu, ce qui peut témoigner d'une légère régression du froid et d'une augmentation de l'humidité. D'après les résultats de l'étude de la microfaune, le paroxysme du climat froid et sec pourrait se situer dans les couches 5 et 6 où les pollens semblent en revanche indiquer une amélioration climatique.

\section{Second secteur}

L'autre secteur de fouilles se situe à quelques mètres au sud-est du précédent en contrebas d'un banc rocheux creusé de carités détruites par d'anciennes carrières. La fouille a concerné la partie haute du talus. La stratigraphie était la suivante :

la, terre végétale riche en racines et en débris organiques et qui contient quelques vestiges paléolithiques 
issus d'anciens remplissages d'abris détruits (ép. : 20$25 \mathrm{~cm})$;

lb, limon brun foncé à granules et petits éboulis émoussés dans un sédiment faiblement humique à structure grumeleuse (ép. : $15-30 \mathrm{~cm}$ ) ;

lc, lit de déblais de carrière peu épais, formant des lentilles ;

1d, limon brunâtre, renfermant des tessons mêlés à des vestiges paléolithiques en position secondaire; un lit de gros bloc discontinu traverse cette couche dont la couleur s'éclaircit à la base au contact des dépôts pléistocènes ;

2a, limon sableux brun clair paurre en granules calcaires et presque sans éboulis, dans une matrice concrétionnée ou ameublie par places par des terriers (ép. : $20 \mathrm{~cm}$ ) ; les vestiges archéologiques sont sporadiques ;

$2 \mathrm{~b}$, limon sableux brun clair, plus riche en granules calcaires et en vestiges que $C 2$ a, mais de même structure et même texture (ép. : $20 \mathrm{~cm}$ ) ;

2c, éboulis de dimensions moyenne dans une matrice limono-sableuse brun clair plus ou moins induree identique à celle de $2 \mathrm{a}$ et $2 \mathrm{~b}$; les vestiges archéologiques son relativement abondants (ép. moyenne : $20 \mathrm{~cm}$ ) ;

3 , limon sableux brun-jaune foncé concrétionné par endroits, riche en granules calcaires et petits éboulis ainsi qu'en vestiges archéologiques (faune et industric lithique), le sédiment devient plus argileux en profondeur. Deux dates ont été obtenues pour cette couche dont nous n'avons pas atteint la base : Ly $2681=15700$ $\pm 450 \mathrm{BP}$ et Ly $4222=15070 \pm 270 \mathrm{BP}$.

\section{Les industries}

Les industries lithiques et osseuses de ce secteur de fouilles présentent de grandes similitudes avec celles du secteur 1 tant en ce qui concerne le choix des matic̀res premières, les caractéristiques technologiques et la composition de l'outillage pour l'industrie lithique que la faible diversification typologique et la fragmentation des pièces pour l'industrie osseuse, et l'on note l'absence d’objets caractéristiques du Magdalénien supérieur et final.

\section{La faune}

La faune associée aux couches magdaléniennes est plus fragmentée dans les niveaux supérieurs (C2a, C2b) que dans les niveaux profonds où elle est également plus abondante. Elle comporte des restes d'antilope saïga, cheval, bison, renne et témoigne de conditions steppiques qui évoquent les conditions du Dryas ancien.

Nous n'avons pas découvert d'objets d'art mobilier dans les deux secteurs tandis que les éléments de parures (coquillages fossiles miocènes et pléistocènes percés) sont bien représentés.

\section{Observations générales}

D'après les données des fouilles récentes, l'occupation magdalénienne du Roc de Marcamps semble se rattacher au Magdalénien moyen. Il existait sans doute des niveaux de Magdalénien supérieur dans le remplissage de la grotte des Fées et probablement aussi dans la partie haute du talus (Lenoir, 1983), immédiatement au pied de la grotte des Fées et plus à l'est où un niveau de brèche renfermant un peu d'industrie et des restes osseux a été daté (Ly $3148: 11910 \pm 230 \mathrm{BP})$. Ce site devait comporter différents locus étagés sur la pente. Les relations entre ces différents locus, et notamment entre le remplissage de la grotte des Fées et les dépôts du talus, demeurent difficiles à établir, le gisement ayant été tronqué par des exploitations de carrière et par les fouilles anciemnes. Les fouilles modernes n'ont pas permis de décourrir de navettes en stratigraphie et de confirmer ainsi l'attribution récente des industries du Roc de Marcamps au Magdalénien à navettes (Allain et al., 1985). Les industries du Roc de Marcamps diffèrent de celles des niveaux supérieurs du talus de Saint-Germainla-Rivière tout proche par la rareté des lamelles scalènes, et les différences sont plus accusées encore arec les industries des niveaux profonds de ce dernier gisement qui cependant semblent appartenir au Dryas ancien. Bien que peu expressives, les industries des couches (i4, C5, C6 du Roc de Marcamps différent peu de celles des niveaux sus-jacents, mais elles pourraient être contemporaines du Badegoulien ou du Solutréen bien caractéristiques et bien datés dans d'autres gisements.

\section{SAINT-GERMAIN-LA-RIVIÈRE}

\section{Historique et description du site}

Ce gisement est situé à Saint-Germain-de-la-Rivière en rive droite de la Dordogne, un peu en aral de sa confluence avec l'Isle. C'est un vaste abri sous roche en majeure partic effondré, creusé dans une haute falaise de 

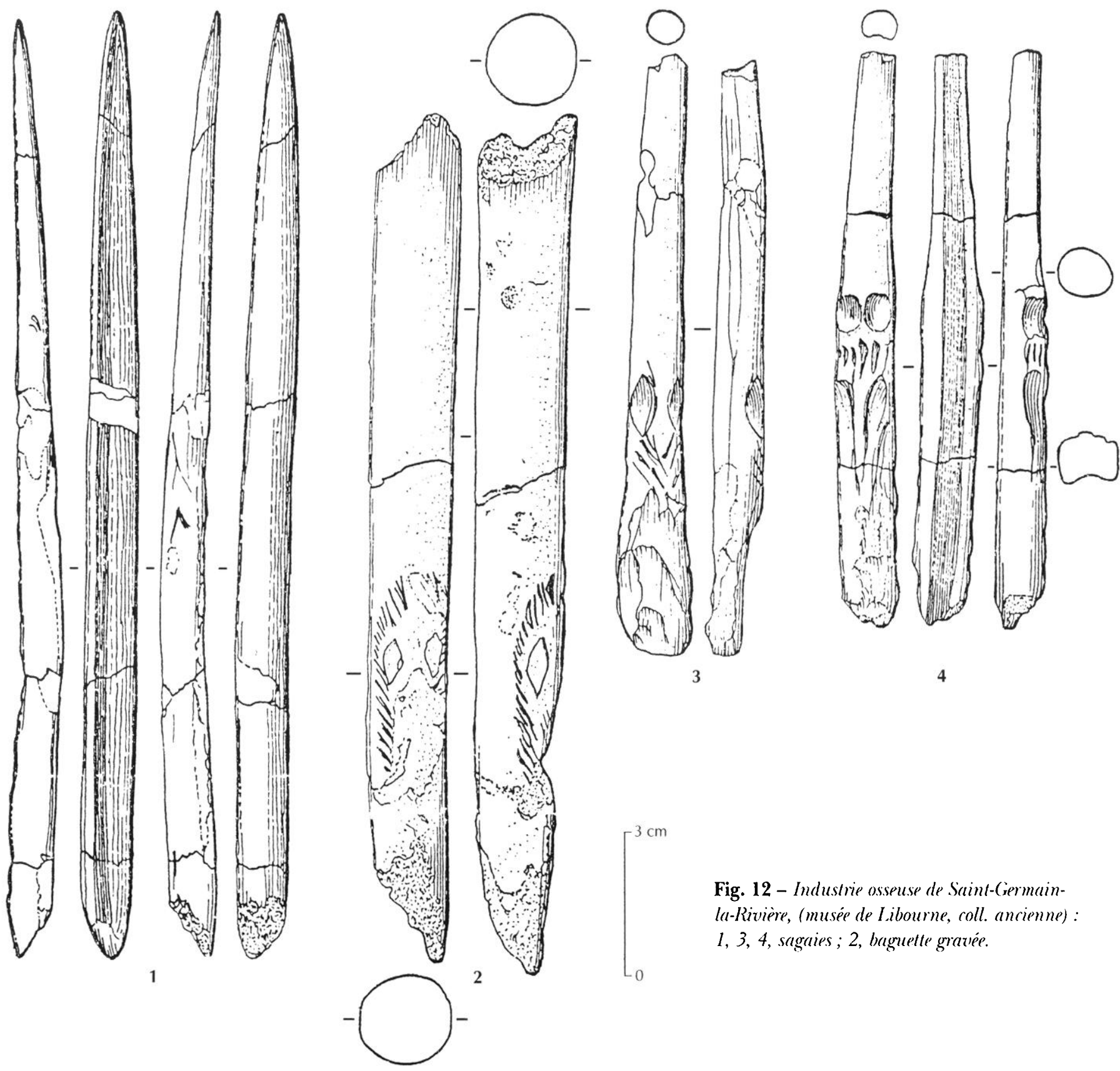

Fig. 12 - Industrie osseuse de Saint-Germainla-Rivière, (musée de Libourne, coll. ancienne): $1,3,4$, sagaies; 2 , baguette gratiée.

calcaire à Astéries. Un talus à pente vive, accidentée de replats, le prolonge et rejoint la plaine alluviale de la Dordogne que le gisement domine. Une cavité effondrée s'ouvre à quelques mètres à l'est. L.e sol rocheux de l'abri principal qui remonte dans sa direction semble former une cuvette dont le fond est constitué par l'argile stampienne. Le gisement a été découvert en 1929 par H. Mirande qui y entreprit des recherches avec R. Lépront. Un premier squelette humain gisant à fleur de terre fut exhumé en 1930 en pied de falaise, mais il tomba en poussière et n'est connu que par quelques fragments crâniens. En 1933, un industriel, R. Blanchard, entreprit des fouilles intensives dans le gisement et il y découvrit en 1934 la fameuse sépulture un peu à l'est du squelette précédent. (Blanchard, 1935, 1937 ; Blanchard et al., 1972).

Deux terrasses séparées par un " magma " de grands ossements d'herbivores furent distinguées par les fouilleurs, la terrasse inférieure qui est celle du grand abri et la terrasse supérieure où furent découverts les 
squelettes. Une seule couche archéologique a été signalée sur la terrasse supérieure et deux couches séparées par un mince niveau d'interoccupation l'ont été sur la terrasse inférieure. La plupart des vestiges recueillis par R. Blanchard et ses notes de fouilles ont disparu dans un incendie. Une faible partie de matériel archéologique a pu être épargnée et elle est conservée au musée national de Préhistoire, aux Eyzies-de-Tayac. Le matériel des fouilles de Mirande et Lépront est en grande partic conservé au musée de Libourne (fig. 12). D. Peyrony a cependant donné une description des industries recueillies par $\mathrm{R}$. Blanchard dans la monographie consacrée au gisement (Peyrony, in Blanchard et al., 1972).

\section{Les industries}

La couche inférieure du grand abri a livré une industrie peu élégante avec des outils sur lames courtes, larges et épaisses ou sur éclats. L'outillage commun, grattoirs, burins dièdres et burins sur troncature retouchée, outils composites, perçoirs, lames à dos, est associé à des lamelles à dos épais, des lamelles à dos denticulées, des lamelles scalènes, des lamelles scalènes denticulées et peut-être quelques vrais triangles scalènes. L'industrie osseuse comporte des sagaies bipointes de section circulaire ou ovalaire, des sagaies à biscau simple ou double, une baguette ornée de traits transversaux parallèles, une portion de côte rainurée. Cette couche fut attribuée au Magdalénien II, compte tenu de la présence de triangles scalènes, mais cette attribution n'a plus guère de signification. La couche supérieure paurre en outils sur éclats comporte surtout des outils sur lames courtes (grattoirs, burins variés, grattoirs-burins, lames retouchées, perçoirs) associés à des lamelles à dos, parfois tronquées. L'industrie osseuse est plus abondante que dans la couche précédente, avec des objets à rainures, des poinçons, des aiguilles à chas, des ciseaux, des sagaies à biseau simple ou double, des bâtons percés, des objets portant des traits gravés obliques, parfois croisés, formant des lignes brisées, d'autres à motifs ovalaires ou triangulaires, un bois de renne à figuration schématique interprétée comme une tête de bison stylisée, une pièce cassée phalliforme sculptée en ronde bosse. L'industrie de la terrasse supérieure se caractérise par un débitage plus soigné avec de grandes et belles lames brutes ou transformées en outils. L'outillage commun comporte les types habituels outre la présence de serpettes de
Loubressac. Les lamelles à dos sont à dos rectiligne épais, accompagnées de quelques lamelles à dos tronquées et de quelques lamelles scalènes. L'industrie osseuse peu abondante comporte des poinçons, de fines aiguilles à chas, un bâton percé sculpté et une grande sagaie ornés de motifs en relief, un fragment de sagaie à base à double biseau, un fragment mésial de sagaie portant des motifs en forme de $\mathrm{V}$, une sagaie biconique complète à profonde rainure longitudinale, un fragment d'outil à double rang de barbelures mal dégagées interprété comme un "proto-harpon ». Le squelette de la sépulture était associé à des éléments de parure (dents et coquillages percés). L'industrie de cette couche et celle de la couche supérieure de la terrasse ont été attribuées par D. Peyrony au " Magdalénien III ".

\section{Les résultats des fouilles récentes}

En 1959, (a. Trécolle a repris, à partir de la tranchée de Mirande, des fouilles dans le talus qui prolonge la terrasse du grand abri. Sous d'anciens déblais de carrière la stratigraphie est la suivante :

C, sable argileux brun-gris, concrétionné, paurre en éboulis et en granules (ép. : 15-20 cm) ;

Cl, sable limoneux brun-gris foncé à passées charbonneuses et petits éboulis et granules calcaires ; concrétionnée dans sa partie supérieure, cette couche devient plus cryoclastique en profondeur (ép.: $87 \mathrm{~cm}$ ), trois lignes cendreuses y ont été individualisées ;

C2, sable limoneux brun gris à éboulis altérés et granules calcaires friables (ép. : $13 \mathrm{~cm}$ ) ;

C.3, sable limoneux brun foncé, à éboulis altérés et granules calcaires friables, reposant par endroits sur l'argile stampienne et qui montre trois lignes cendreuses;

C4, couche cryoclastique localement représentée entre (.3 et le substratum, à éboulis calcaires peu volumineux, relativement émoussés, associés à des granules calcaires dans une matrice sablo-limoneuse brun-gris.

Deux ensembles industriels ont été distingués dans cette séquence. Le premier, inférieur, concerne les couches (.4, C.3 et dans une certaine mesure (.2, le second, supérieur, concerne Cl et C. (Lenoir et al., 1991, $1994,1995)$.

L'ensemble inférieur se caractérise par une industrie peu laminaire, riche en éclats épais, pour la plupart corticaux avec de nombreuses entames. Les lamelles sont bien représentées ainsi que les nucléus. Les éclats sont à 
talon lisse épais, de section triangulaire et portent de larges plages de cortex. Ils ont été détachés au percuteur de pierre selon un mode de débitage apparemment peu organisé qui consiste à débiter le nucléus en changeant sans cesse de plan de frappe, de telle sorte que deux enlèvements successifs ont des directions plus ou moins orthogonales. Ce mode de débitage permet d'obtenir des éclats épais qui ont pu servir de support à des outils mais plus fréquemment ont été transformés en nucléus à lamelles. Les lamelles ont été détachées transversalement ou latéralement par rapport à l'axe d'allongement de l'éclat à partir d'un plan de frappe constitué par un pan naturel (négatif dorsal ou méplat latéral) ou d'une troncature retouchée épaisse. Ces lamelles, à talon lisse, sont pour la plupart larges, courtes et de profil légèrement incurvé, parfois torses. Certaines peuvent aroir été obtenues à partir de nucléus carénés ou de grattoirs épais. Les plus fines ont été transformées en lamelles à dos d'un type particulier, à dos plus ou moins rectiligne, à fine retouche marginale opposée à un bord brut convexe. Cette industrie peu laminaire, associant de petites lamelles à dos à un outillage peu diversifié, comportant des outils généralement épais, sur éclats, se distingue de celle des niveaux sus-jacents dans le talus et des couches représentées dans les abris. Elle ne présente pas les caractéristiques du Badegoulien et diffère des industries habituelles du Magdalénien moyen ou du Magdalénien supérieur. Ses caractéristiques particulières résultent peut-être de ce qu'elle est spécialisée dans la production des microlamelles à partir de nucléus sur éclats épais.

Les couches supérieures du talus ont livré des industries bien différentes de la précédente et qui rappellent celles du remplissage du grand abri. Elles sont nettement plus laminaires et demeurent lamellaires et les éclats corticaux épais sont bien moins représentés. Le débitage laminaire présente les caractéristiques d'un débitage magdalénien classique avec présence de lames à crête, de tablettes de ravivage de plans de frappe de nucléus qui comportent des exemplaires prismatiques à un seul plan de frappe ou à deux plans de frappe opposés, outre des nucléus à éclats devenus informes par exhaustion. Les lames de bonne facture ont pour la plupart servi de pièce-support à l'outillage constitué de grattoirs et de burins. Les lamelles sont plus régulières et mieux standardisées que dans l'ensemble inférieur, avec des bords et des nerrures parallèles et elles sont souvent transfor- mées en lamelles à dos épais et abrupt associées à des lamelles scalènes et des lamelles à dos denticulées.

L'industrie osseuse est la même dans les deux ensembles. Elle comporte des outils pour la plupart fragmentaires, aiguilles à chas, sagaies à section ovalaire ou quadrangulaire parfois à rainures, baguettes et os façonnés.

Les résultats d'une étude sédimentologique effectuée sur les dépôts du talus (Lenoir, 1970) méritent d'être reconsidérés selon de nouveaux critères et les méthodes d'étude actuelles. Ceux de l'étude palynologique de M.-M. Paquereau (Lenoir, Paquereau, 1986) ont révélé des flores peu boisées à caractères steppiques qui s'atténuent un peu au sommet de la couche $C$. Les associations fauniques sont très semblables d'un niveau à l'autre, avec prédominance de l'Antilope saïga. Elles traduisent les conditions très rigoureuses et steppiques qui régnèrent en Aquitaine au cours du Dryas ancien (Ouzrit, 1986). Les résultats d'une étude archéozoologique concernant les faunes recueillies par G. Trécolle dans le talus (Costamagno, 1999) indiquent " une occupation temporaire consacrée à l'exploitation des carcasses chassées d'un point de vue nutritif plutôt qu'un camp résidentiel de longue durée ".

Des travaux de terrain récents (en 1996) nous ont permis de déterminer l'étendue des fouilles anciennes. Le grand abri semble avoir été presque complètement vidé de son remplissage dont il reste peut-être un témoin sous un gros bloc effondré qui en occupe la partie centrale. Les dépôts encore visibles dans la partie est de cet abri sont apparus remaniés (déblais de fouilles ou déblais de carrière anciens). Le " magma " à gros ossements d'herbivores signalé par les anciens fouilleurs à la limite des terrasses des deux abris semble avoir disparu. Il ne subsiste plus que sous forme de sédiments remaniés dont une partie peut être issue de la vidange de cavités naturelles détruites par d'anciennes galeries de carrière. Les dépôts qui reposaient sur le plancher de l'abri supérieur ont été détruits presque en totalité. Dans le talus, de part et d'autre de la tranchée de fouilles anciennes, subsistent des couches riches en industrie et en faune. La succession stratigraphique des deux ensembles d'industries distingués par G. Trécolle a été confirmée par le ravivage de la paroi est de la tranchée qui montre des dépôts apparemment soliflués, mais qui ne semblent pas avoir subi un grand déplacement. Les couches profondes paraissent moins perturbées que celles du sommet. 
Tabl. I - Datations obtemues sur des os provenant des dépôts du talus de lalmi de Saint-Germain-la-Riviere.

\begin{tabular}{|l|}
\hline C GIF $6037: 14100 \pm 160 \mathrm{BP}$ \\
\hline C1 OXA 7345 / Lyon $615: 15330 \pm 150 \mathrm{BP}$ \\
\hline C2 GIF $5478: 15300 \pm 410 \mathrm{BP}$ \\
\hline C3 OXA 7 259 / Lyon 616:2385 $\pm 45 \mathrm{BP}$ \\
\hline C4 GIF $6037: 14100 \pm 160 \mathrm{BP}$ \\
\hline "Magma d'os »: OXA 7258 / Lyon $614: 15510 \pm 120 \mathrm{BP}$ \\
\hline
\end{tabular}

Nous disposons pour ces dépôts du talus de datations effectuées sur des os recueillis lors des fouilles de G. Trécolle et lors des travaux récents (tabl. I).

\section{LES AUTRES GISEMENTS DU DRYAS ANCIEN}

Au cours du Dryas ancien ont été occupés de petites cavités ou des talus en pied de falaises en rive droite de la Dordogne: Fongaban à Saint-Émilion ; dans l'EntreDeux-Mers occidental : l'abri Houleau sur la vallée de la Gamage, les abris de Jaurias (Cousté, 1963) et de Moulin-Neuf dans le bassin de la Canodonne et les niveaux inférieurs de la grotte de Fontarnaud à Lugasson dans la vallée de l'Engranne (Labrie, 1928), tandis que la vallée de la Garonne n'a pour l'instant livré qu'un gisement: la grotte de l'Ermitage à Bouliac près de Bordeaux (Magne, 1940 ; Cousté, 1948 ; Lenoir, Roussot, 1994). Les Magdaléniens y ont abandonné des industries laminaires et lamellaires associant aux lamelles à dos abondantes un outillage commun où les burins dièdres dominent les grattoirs. L'industrie osseuse dépourvue de harpons comporte des sagaies fusiformes de section ovalaire ou circulaire, des sagaies de section quadrangulaire avec ou sans rainure médiane et à biseau, outre des baguettes, des poinçons, des aiguilles à chas, des bâtons percés, des navettes au Roc de Marcamps (Allain et al., 1985). Dans la faune, l'Antilope saiga domine, accompagnée du Renne du Cheval et du Bison très abondant à Fongaban (Delpech in Rigaud et al., 1972). Un gisement (Jaurias) a livré des pièces à cran d'un type particulier (Cousté, 1956, 1963; Desbrosse, Kozlowski, 1988, 1989 ; Lenoir, 1983) attribuées au Magdalénien supérieur (Sonneville-Bordes, 1988) mais qui appartiennent très probablement au Magdalénien du Dryas ancien.

\section{LE MAGDALÉNIEN RÉCENT ET LE PASSAGE À L'AZILIEN}

Au cours du Dryas moyen, en Gironde, les conditions climatiques furent moins sévères et moins steppiques que pour le Dryas ancien. Dans des paysages de prairies à graminées prédominantes (Paquereau, in Lenoir, Paquereau, 1986) se développent des faunes à Cheval, Renne, grands bovidés, Cerf, Harfang et Antilope saïga. (Delpech, 1975, 1979, 1983). Les Magdaléniens occupent des cavités (grotte de Fauroux et grotte de Fontarnaud à Lugasson, abri Vidon à Juillac, abri Morin à Pessacsur-Dordogne, La Pique à Daignac) mais probablement aussi des gisements de plein air sur des replats de versant ou en pied de falaise, plus difficiles à dater. Les industries lithiques sont laminaires et lamellaires avec un fort pourcentage de lamelles à dos épais et l'apparition d'outils nouveaux et d'armatures: pointes à cran magdaléniennes (Lenoir, 1975), pointes de Laugerie-Basse (Bordes et al., 1973), pointes aziliennes (Bordes, Sonneville-Bordes, 1979), lames tronquées et usées (Lenoir, Terraza, 1979), lames retouchées magdaléniennes, scies (Deffarge, Sonneville-Bordes, 1972), grattoirs courts, burins de Lacan (Labrie, 1904) et burins bec-de-perroquet. L'industrie osseuse témoigne également d'innovations avec l'apparition des harpons de section cylindrique à un ou deux rangs de barbelures plus ou moins dégagées associés à des poinçons, des ciseaux, des lissoirs et des aiguilles (Deffarge et al., 1974a, 1974b, 1977). Quelques gisements (abri Morin, abri Faustin à Cessac et grotte de Fontarnaud) sont connus notamment pour avoir livré des ceuvres d'art mobilier (Labrie, 1928 ; Roussot, Ferrier, 1971 ; Deffarge et al., 1975 ; Lenoir, 1983). Au cours des toutes dernières années, l'extension des recherches dans le secteur compris entre la plaine alluviale de la Garonne et l'océan y a révélé une présence magdalénienne (Lenoir et al., 1997 ; Belbeoc'h, Lenoir, 1996, 1998). Les fouilles en cours du gisement de plein air de La Honteyre au Tuzan, dans la couverture sableuse qui porte la forêt landaise, concernent un petit habitat du Magdalénien supérieur qui n'a conservé que l'industrie lithique (fig. 13). Cette industrie est associée à des apports de grès ferrugineux qui pourraient avoir servi de pierres de foyers ou pour assainir le substratum. Le débitage est laminaire et lamellaire, riche en lamelles à dos épais. L'outillage commun, où les burins dominent les grattoirs, comporte des burins pour la plupart dièdres ou 


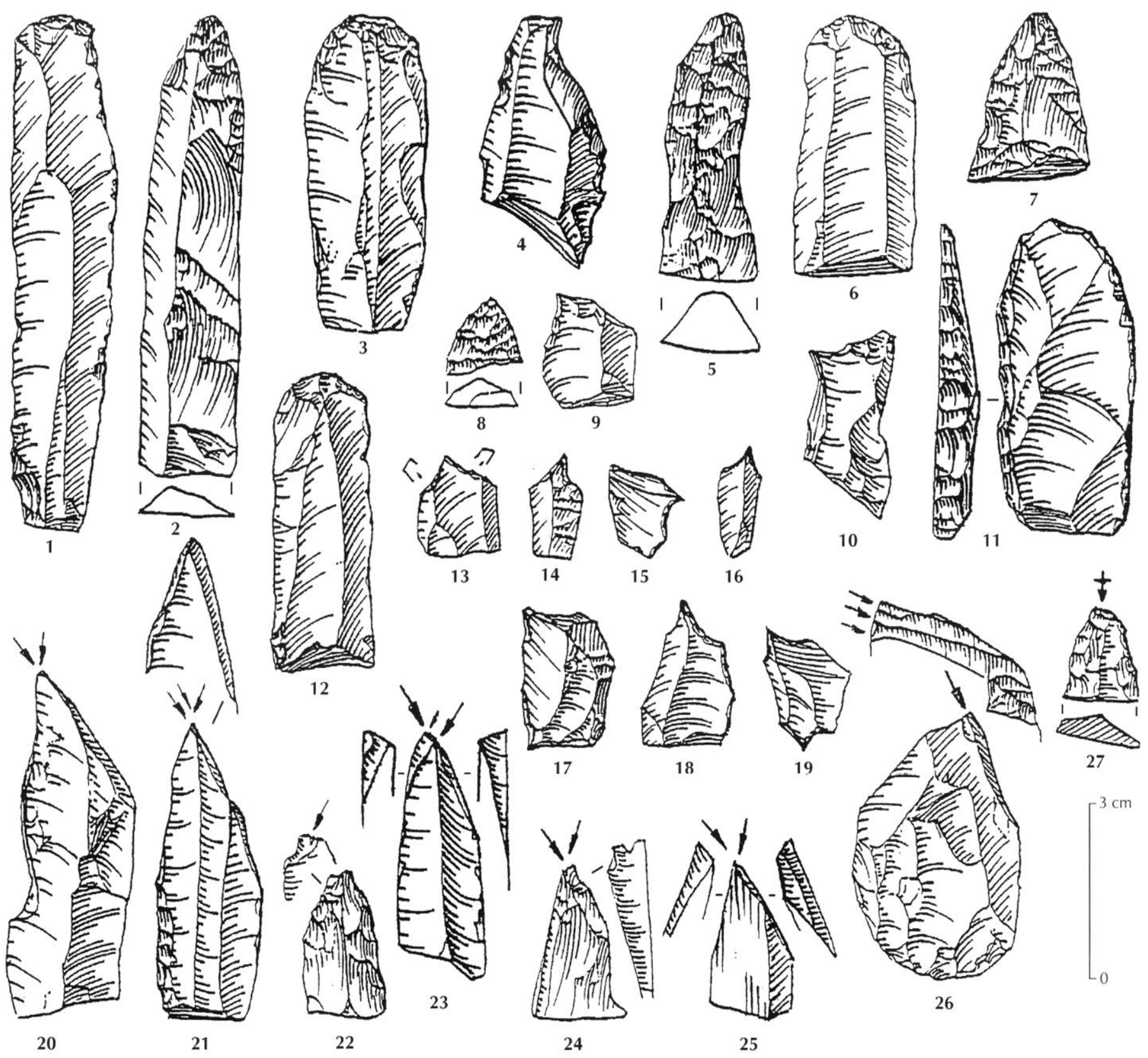

Fig. 13 - Industrie lithique du Magdalénien supérieur de La Honteyre, Le Tuzan : 1-3, 5-7, 8, 11, 12, grattoirs; 4, denticulé; 9, 10, 13-19, persoirs; 20-26, burins; 27, fragment de lame retouchée.

sur cassure, trois burins bec-de-perroquet, des perçoirs et des microperçoirs, des lames tronquées, des fragments de lames retouchées. Les silex semblent pour la plupart allochtones. Certains proviennent d'alluvions, ainsi qu'en témoigne l'usure des plages corticales, où ils ont été recueillis sous forme de petits blocs dans des gîtes primaires de silex sénonien. Ils sont associés à des fragments de galets de quartz ou de quartzite, éclatés par le feu. Les restiges organiques ne se sont pas conservés dans ces milieux sableux et humifères. Le silex campanien du bombement anticlinal de Villagrains, tout proche, semble avoir été peu exploité et il en est de même des silex aquitaniens lacustres du Bazadais.

Avec l'instauration des conditions plus douces et plus humides de l'Alleröd, le milieu animal et végétal va montrer des changements et il en est de même de la culture 
matérielle. Les harpons plats font leur apparition, le débitage laminaire devient moins élaboré, l'outillage se charge en grattoirs courts et en pointes à dos et l'on voit apparaître plus nettement les microlithes géométriques. Dans la région calcaire interne (fig. 10), l'habitat concerne de modestes cavités, de petits gisements en pied de falaise, tandis qu'à l'ouest, entre Garonne et océan, sont connues de petites stations en bordure de crastes et de points d'eau (Roussot-Larroque, 1979; RoussotLarroque, Callède, 1976). La chasse concerne désormais des herbivores de milieu forestier (Gilbert, 1984), l'art mobilier devient sporadique (Lenoir, Roussot, 1975) et, succédant à l'épisode froid du Dryas récent, s'instaurent peu à peu les conditions postglaciaires. Des technocomplexes nouveaux, riches en petits éclats et en microlithes géométriques, se substituent à l'Azilien et serviront de substrat à la néolithisation.

\section{LES MODES D'IMPLANTATION AU COURS DU PALÉOLITHIQUE EN GIRONDE}

Les traces les plus anciemnes de présence humaine dans les bassins versants des basses vallées de la Dordogne et de la Garonne demeurent mal connues, très sporadiques et de datation imprécise, puisqu'il s'agit presque exclusivement de produits de débitage peu diversifiés, en silex ou en quartzite, sans restes de faune associés, qui témoignent de l'exploitation préférentielle de matières recueillies en contexte alluvial. Au cours du Riss, probablement dans sa partie terminale, les matières premières siliceuses locales (silex lacustres du (Cénozoïque), peut-être plus accessibles, en position secondaire dans des cailloutis de solifluxion, ont attiré l'homme qui les a exploitées assez intensément, comme en témoignent de nombreux ateliers implantés sur les gîtes, tandis que les vrais habitats demeurent rares et sont difficiles à caractériser parce que trop brefs, remaniés, ou détruits. Les hommes de Néandertal ont occupé des sites de plein air à proximité de gîtes de matières premières siliceuses (dépôts alluviaux ou gîtes de silex local cénozoïque ou campanien), mais ils ont également fréquenté les porches de cavités, comme à Pair-non-Pair ou à Haurets. Les gisements moustériens ou châtelperroniens en grottes ou sous abris demeurent cependant rares en Gironde et c'est dans la période de transition du Würm ancien au Würm récent que l'on connaît plusieurs repaires d'hyènes, qui semblent avoir fréquenté les cavités naturelles en alternance avec l'homme. Quelques indices très sporadiques de Solutréen sont connus dans des stations de plein air, mais la présence de Solutréen est mieux attestée dans le talus du gisement du GrandMoulin, site de la moyenne vallée de l'Engranne au cœur de l'Entre-Deux-Mers, où elle avait été déjà anciennement signalée par J. Labrie. Dans ce secteur l'on connaît également plusieurs gisements magdaléniens en grottes ou sous abris qui prennent le relais de petits sites badegouliens, généralement placés sur des points hauts. Le Dryas ancien apparaît comme un épisode asse $\%$ long, bien marqué dans les séquences stratigraphiques sous abri et qui témoigne par les faunes et les flores de conditions froides et sèches à caractères steppiques au cours desquelles l'Antilope saïga trouve des conditions de vie optimales alliées à un relief peu accidenté, parfaitement adapté à son séjour dans la plaine de Gironde. À la fin des temps glaciaires, dans un contexte du Magdalénien supérieur et final, se développe l'art mobilier et l'on voit apparaitre dans les ensembles lithiques des types d'outils nouveaux, notamment des armatures spécifiques à ces technocomplexes, généralement très laminaires et lamellaires, qui témoignent d'une certaine variété d'approvisionnement en silex. L'Antilope saïga a disparu de ce secteur et les modes de subsistance se diversifient. Les derniers Magdaléniens, qui ont débordé la zone calcaire interne et se sont aventurés dans le secteur situé à l'ouest de la Garomne, ront peu à peu céder la place à des groupes dont la culture matérielle témoigne de leur appartenance azilienne et dont la filiation avec le Magdalénien demeure incontestable. Les indices d'une présence azilienne sont surtout connus dans la Gironde interne, dans des porches de petites cavités, mais on les connaît également dans de modestes stations de plein air proches du littoral actuel et beaucoup plus éloignées du littoral de l'époque. L'art mobilier régresse par la technique et l'esthétique voire même disparaît. Les modes d'approvisionnement en matières premières deviennent moins diversifiés tandis que les faunes sont marquées par la prédominance d'espèces forestières. À ces cultures matérielles, riches en pointes à dos, se substituent les technocomplexes propres au Sauveterrien qui se chargent en armatures microlithes géométriques dans des contextes archéologiques relativement discrets, petites stations de plein air dans les sables, modestes cavités et petits gisements en pied de falaise de la région calcaire. 
Désormais, la chasse des troupeaux de grands herbirores cède le pas à des modes de subsistance plus variés où la part respective de la chasse du gros gibier et de l'exploi- tation de gibier de petite taille et celle de la collecte des ressources végétales restent à établir dans ce secteur de l'Aquitaine. 


\section{BIBLIOGRAPHIE}

Ai.lain J., Desbrosse R., Kozi.owski J. K., Ricilld A.

1985 : Le Magdalénien à navettes, Gallia Préhistoire, 28, 1, p. 37-124.

BeIBEOC'H G., LFNOIR M.

1996 : Données nouvelles sur l'occupation de la Iande girondine, in: Les landes entre tradition el écologie, Actes du colloque de Sabres (Landes), 25-26 mars 1995, Fédération historique du Sud-Ouest, Société de Borda, Parc naturel régional des Landes de (ascogne, p. 63-79.

1998 : Recherches sur l'occupation du sol dans la région dHostens, Travaux et colloques scientifiques, Parc naturel régional des I andes de (aascogne, 2, p. 37-42.

BIAN(HARD) R.

1935 : Découverte d'un squelette humain à Saint-cermain-la-Rivière, Rezue historique et archeologique du Liboumais, 9, p. 11-18.

1937 : Notice sur le fossile humain de Saint(iemmain-la-Rivieve (Gironde), Montignac, I.evmarie imp., 13 p.

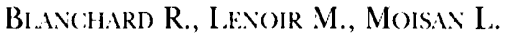

1976: Nouveaux indices du Solutréen en Gironde, Rezue historique at archéologique du Libournais, XI.IV, p. 82-84.

BI.NY(HARD R., PEIRONY D), VIIIOHS H.-V. 1972 : Le gisement et le squelette de SaintGermain-la-Riviere, Archives de l'Institut de paléontologie humaine, mém. 34, Paris, Masson, 115 p.

BORILES F.

1953 : Essai de classification des industries "moustériennes ", Bulletin de la Société préhistorique francraise, L, 7-8, p. $457-466$.

1981 : Vingt-cinq ans après : le complexe moustérien revisité, Bulletin de la Société préhistorique francaise, 78, 3, p. $77-87$.
Bordes F., Deffarges R., SonveitlleBORDES D. DE

1973 : Les pointes de Laugerie-Basse dans le gisement du Morin. Essai de définition, Bulletin de la Société préhiscorique francaise, 70 , crsm 5, p. 145151.

Bordes F., SONATYIIIF-BORIDES D. DE

1979 : Lazilianisation dans la vallée de la Dordogne. Les données de la Gare de Couze (Dordogne) et de l'abri Morin (Gironde), in: SonnevilleBordes D. de (éd.), La fin des Temps glaciaires en Europe. Chronostratigraphie et écologie des rultures du Paléolithique final, 1, Colloques internationaux du CNRS, $\mathrm{n}^{\circ}$ 271, Talence, 24-28 mai 1977, Paris, éd. du CNRS, p. 449-459.

Bol TIt:k J.-M., Rol sstal J.-M.

1972 : Fragment crânien humain d'âge rissien des alluvions de la Dordogne, I.Anthropologie, 76, 3-4, p. 32:5-330.

\section{BRFIII. H.}

1911 : Études de morphologie paléolithique. Lindustrie de la grotte de Chatclperron (Allier) et d'autres gisements similaire, Ronue anthropologique, 1, p. 29-40 et 2, p. 67-76.

Brial. (;., Defrarge R., Pírol. J.-F.

1954 : Première molaire aurignacienne de "Che\% Leix ", Bulletin de la Sociélé d'études et de recherches próhistoriquse des liyzies, $4 \mathrm{p}$.

BRI:Kl:R H. (ED).)

1995: Ie Paléolithique supérienter de lAbri Palaud (I)ordogne). Les fouilles de H.L. Movius fr: Paris, ed. de la Maison des sciences de l'homme, Documents d'archéologie française, 50, $325 \mathrm{p}$.

\section{Chemiler A.}

1963 : La Caverne de Pair-non-Pair; Gironde. Fouilles de François Daleau, Document d'Aquitaine, II, Publication de la Société archéologique de Bordeaux, $213 \mathrm{p}$.
Cosmanaginos.

1999 : Strateggies de chasse et fonction des sites au Magdalénien dans le Sud de la France, thèse de Doctorat, univ: de Bordeaux I, 2 vol., 495 p.

(O)'STÉ R.

1948 : Grotte de l'Ermitage, Le Marais, Gironde, Bulletin de la Société spétéologique et préhistorique de Bordeaux, 1 , p. $6-9$.

1951: Gisement magdalénien des grottes de Jaurias, Bulletin de la Société prénistorique fransaise, XIVIII, 7-8, p. 382-384.

1956 : Sur un niveau de Paléolithique supérieur de la Gironde, Bulletin de la Société préhistorique frangraise, XI.III, 11 12, p. 679-681.

1959a : Les subdivisions du Magdalénien de la (ironde, in: Actes du 32 congrès des sociétés savantes, Bordeaux, 1957 . p. $27-33$.

1959b: Ie Paléolithique dans la basse vallée de la Dordogne, in: Actes du congres ammuel de liAssociation des amis et naturalistes de la vallée de la V'éère, Ie Bugue, 23 aôt 1959,12 p.

1963 : Gisement magdalénien des grottes de Jaurias ì Saint-Quentin-de-Baron ((jironde), Tirage ronéotypé, Bordeaux, 105 p.

\section{Col'sté R., Krtolttza Y.}

1965 : Labri lespaux (Cne de SaintQuentin-de-Baron) et la question du Périgordien en Gironde. Note préliminaire, Revue historique et archeiologique du Libourmais, 116 , p. 1-8.

DNELIC F.

1874 : (rrotte des Fées (âge du Renne) située all Roc, commune de Marcamps, canton de Bourg (Gironde), Société archéologique de Bordeaux, 1, p. 109-119.

1881 : La grotte de Pair-non-Pair à Marcamps, in: Association fransaise pour l'avancement des sciences, $10^{\circ}$ session, Alger, p. 755. 
1910: Silex à retouches anormales de ba station de la Bertonne ou la Rousse, commune de Peujard (Gironde), Sociélé archéologique de Bordeaux; XXXI, p. 3I-48.

DALAL F., GASSIES J.-B.

1874 : Notice sur la station de Jolias, commune de Marcamps (Gironde), Rerue d'anthropologie, III, p. 470-477.

D.IIII) N. C:

1966: The Perigordian Vo: an upper paleolithic culture in Western Europe, PHD Thesis, Harvard University, Cambridge Massachussets, LSA, $755 \mathrm{p}$.

1985: Exatuations at the Abri Pataud, Les liyzies (I)ordogne). The Noaillian (level 4) assemblages and the Noaillian culture in Western Europe, American School of Prehistoric Research, 37, 355 p.

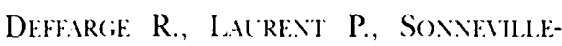
BORIDES D. DE:

1974a: I.es harpons de l'abri Morin (commune de Pessac-sur-Dordogne, (Cironde), in : Premier colloque internalional sur l'Industrie de l'os dans la Próhistoire, Abbaye de Sénanque, 1974, p. 193-218.

1974b) : Ciseaux ou lissoirs magdaléniens, Bulletin de la Socióte préhistorique fran(aise, 71, crsm 3, p. 85-96.

1975 : Art mobilier du Magdalénien supérieur de labri Morin à Pessacsur-Dordogne (Gironde), Gallia Préhistoire, 18, 1, p. 1-64.

1977 : Sagaies et ciseaux du Magdalćnien supérieur du Morin, Gironde, un essai de définition, in: Méthodologie appliquée à l'Industrie de l'os préhistorique. Abbave de Sénanque, 1976, Colloques internationaux du C.NRS, n"568, p. 99-110.

DefFarge. R., SoNineth IF-Bordes D. DE 1972 : Ia scie, fossile directeur lithique du Magdalénien final, Bulletin de la Socielé préhislorique francaise, 69, crsm 5. p. 140-144.

DFI.I'T:CH F.

1975: L.es faunes du Paléolithique supérieur dans le Sud-Ouest de la France, thèsc de
Doctorat d'Étal ès sciences, unis. Bordeaux I, $374 \mathrm{p}$.

1979 : Les faunes de la fin des temps glaciaires dans le Sud-(onest de la France, in: Sonneville-Bordes D), de (éd.), la fin des Temps glaciaires en Europe. Chronostratigraphie et écologie des cultures du Paléolithique final, 1, Colloques internationaux du CNRS, $\mathrm{n}^{\circ}$ 271, Talence, 24-28 mai 1977, Paris, éd. du (NNRS, p. 169-175.

1983 : Les faumes du Paléolithique supérieur dans le Sud-Ouest de la France, Paris, éd. du CNRS, Cahiers du Quaternaire, 6, $453 \mathrm{p}$.

DESBROSSE: R., KoZI.OW'SKI J. K.

1988 : Le Paléolithique final entre Atlantique et Vistule. Comparaisons entre les civilisations de la plaine et celles des plateaux, in : Otte M. (éd.), De la Loire a l'Oder: Les civilisations du Paleolithique final dans le .Vord-Ouest européen, Actes du colloque de Liège, déc. 1985, BAR, International Series, 444,1 , p. $655-681$.

1989 : Les origines du "CreswelloHambourgien ", L'Anthropologie, 93, 1, p. 183-188.

DI'BRELIL.H J.

1976: Contribution à l'étude sédimentologique du système flurvatile Dordogne Garonne dans la région bordelaise, thèse de Doctorat d'Université, univ. de Bordeaux, $273 \mathrm{p}$.

Espakza Sax Jian X.

1995 : La curva de Isturitz y su relacion con la cormisa cantabrica durante el Paleolitico superior, Madrid, Universidad nacional de educaciòn a distancia, $309 \mathrm{p}$.

FERRILR J.

1938 : La Préhistoire en Gironde, I.e Mans, Monnoyer imp., $336 \mathrm{p}$.

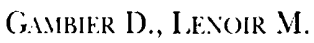

1991 : Les restiges humains du Paléolithique supérieur en Cironde, Bulletin de la Société d'anthropologie du Sud-Ouest, XXXVI, 1, p. 1-31.

GII BERT A.

1984 : Contribution ci l'étude des faumes de la fin des temps glaciaires et du début des temps postglaciaires, these de Doctorat d'Lniversité, univ: de Bordeaux I, $322 \mathrm{p}$.

G(AD)HLI.I J.L

1987 : Contribution ì l'ótude des zoocénoses préhistoriques en Aquituine (Würm ancien et interstade zuïrmien), thèse de Doctorat, univ: de Bordeaux I, $568 \mathrm{p}$.

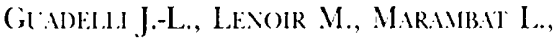
PAQLEREAC M.-M.

1988 : Un gisement de l'interstade Würmien en Gironde: le gisement de Camiac à Camiac-et-Saint-Denis, in: I.homme de Vúandertal, -4- La Technique, Actes du colloque international de Liège, 1986, Études et recherches archéologiques de l'université de Liège, 31, p. 39-69.

KRTOLIT\%A Y., L.F.NOIR M.

1998 : Ln gisement de Gravettien à burins de Noailles de (Gironde : l'abri Lespaux (Saint-Quentin-de-Baron), Revue archéologique de Bordeaux, I.XXXIX, p. 47-68.

L.IBRIF J.

1904: Ln noureau type de grattoirburin: observations complémentaires, Rerue de l'Ecole d'anthropologie de Paris, 54, p. 53-54.

1905: La caverne préhistorique de Haurets à Ladaux, Procès verbaux de la Société linnéenne de Bordeaux, LX, p. CVIII-CXI.

1923 : L.es cavernes et abris préhistoriques de l'Entre-Deux-Mers (Gironde), Association française pou l'avancement des sciences, $47^{\circ}$ session, Bordeatux, p. 657-663.

1928 : Ia caverne préhistorique de Fontarnaud à Lugasson (Gironde), Reoue historique de Bordeaux et du département de la Gironde, 21, p. 102-113.

LACORRE. F.

1938 : I a grotte des Fés à Marcamps (Gironde) ou Roc de Marcamps (fouilles du groupe de Prchistoriens de la Société Linnénne), Procies verbaux de la Sociélé linnéenne de Borderaux, $\mathrm{X}(:$, p. 3i)-isl. 
I FGIGAN P., I.F.NOIR M.

1990 : Exemples d'ateliers de transformation du silex : les sites préhistoriques de la ride anticlinale de Villagrains (Gironde), in: Le silex de sa genèse à l'outil, Actes du V" colloque international sur le silex, Bordeaux, 17 sept.-2 oct. 1987, Cahiers du Quaternaire, 17, p. 429-432.

Legigan P., LeNoIR M., Rigal:D J.-P.

1978 : Découverte d'industrie lithique dans les alluvions quatemaires de la vallée du Gua-Mort (Girondc), L.es Cahiers du Bazadais, p. 3-26.

\section{LENOIR M.}

1970 : Recherches sédimentologiques concernant quelques gisements magdaléniens de Guyenne occidentale, thèse de Doctorat en géologie, univ. de Bordeaux, $129 \mathrm{p}$.

1974 : Indices de Solutréen en Gironde, Bulletin de la Société préhistorique francaise, 71, crsm 7, p. 200)-201.

1975: Observations sur les pointes à cran magdaléniennes dans les gisements de l'abri Faustin, commune de Cessac (Cironde) et de la Pigue, commune de Daignac, (iononde, Bulletin de la Sociéti próhistorique framcaise, 72, crsm 4, p. 107-112.

1976 : Étude typologique et technique des "pièces à retouches anormales" de la station de la Bertonne, commune de Peujard (Gironde), Bulletin de la Société préhistorique fransaise, 73, crsm 2, p. 43-47.

1977 : Un gisement de plein air du Périgordien supérieur en (ironde: les Artigaux à (amiac-et-Saint-1)enis, Bulletin de la Société préhistorique fran(aise, 74, ct. ct trav. 2, p. 518-530.

1980 : Fouilles de sauvetage dans un gisement du Pléistocène supérieur en Gironde : le gisement de la cimenterie d'Espiet, à Camiac-et-Saint-Denis (Gironde), Reque historique et archéologique du Libournais, XIVIII, 176 , p. 41-51.

1982 : I.e gisement du (rand-Moulin à Lugasson (Gironde). Quelques nouvelles observations, Bulletin de la Société préhistorique fransaise, 79, 1 , p. $28-32$.
1983 : Le Paléolithique des basses vallées de la Dordogne et de la Garomne, thèse de Doctorat d'État ès Sciences, univ. de Bordeaux I, 2 vol. 702 p.

1985 : Présence humaine dans la Grande Lande au Paléolithique et à L'Épipaléolithique, in : La cirande Lande. Histoire naturelle et géographie historique. Actes du colloque de Sabres (Landes), 27-29 nor: 1981, Paris, éd. du C.NRS, p 83-95.

1986 : La Préhistoire de Ladaux, in : ì la découverte de l'Entre-Denu-Mers. Ladaux, A.S.P.E.C.T, p. 15-20.

1988 : Lc Magdalénien ancien en Gironde. Conditions de gisement, variablité typologique et technique, in: Lpper Pleistocene Prehistory of Western Eurasia, The Lniversity Museum, University of Pennstlvania, Lniversity Museum Monograph 54, University Museum Symposium Series, vol. 1, Philadelphia, p. $397-410$.

1990) : Ie passage du Paleolithique moven au Paleolithique superieur dans les basses vallees de la Dordogne et de la baronne, in: Paléolithique moven récent et Paléolithique

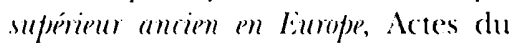
colloque international de Nemours, 9-11 mai 1988, Mémoires du musée de Préhistoire d'Ile de France, 3, p. $21: 5-221$.

1993a: Un gisement magdalénien en Gironde: le Roc de Marcamps à Prignac-et-Marcamps, Bulletin de la Société linnéenne de Bordeaux, 21, 2, p. 75-85: 21, 3, p. 87-108; 21, 4, p. $131-145$.

1993b: Ie gisement du Roc de Marcamps (Prignac-et-Marcamps, (Gironde), Les Ciahiers du Vitrezais, 85-86, p. 1-13.

LeNolR M., Marmitr F., Trécollie (i.

1991 : Données nouvelles sur les industries de Saint-Cermain-la-Rivière (Gironde), in : 25 ans d'études technologiques en Préhistoire. Bilan et perspectives, Actes des rencontres 18-20 octobre $1990, \mathrm{XI}^{\circ}$ rencontres internationales d'Archéologie et d'Histoire d'Antibes, Juan les Pins, éd. ADPCA, p. $245-254$.
1994 : Lce gisement magdalénien de Saint-Germain-la-Rivière (Gironde) : données anciennes et acquis récents, Revue archéologique de Bordenux, I.XXXV, p. 71 .

1995 : Le gisement paléolithique de Saint Germain-la-Rivière (Gironde), Soriété d'anthropologie du Sud-Ouest, XXX, p. 134-153.

L.NOIR M., Obry J., SHRONIE-VIVIEN M.-R. 1997: Occurence of allochtonous flint in an Lpper Paleolithic assemblage near Bordeaux, in : Silicous Rocks and Culture, Actes du $6^{\text {th }}$ International flint symposium, Granada, 1997, p. $385-390$.

I.NOIR M., P.AQLEREAL M.-M.

1986 : Milieux et cultures de la fin du Wïrm en Gironde, Arqueologia, 13, volume en hommage à Jean Roche, p. $31-6.5$.

I.FOIR M., Rotssot A.

1975: Line pendeloque en os décorce de la grotte du Roc à Saint-Sulpice-de(inilleragues (Gironde), Bulletin de la Société próhistorique franşaise, 72, crsm 8, p. $24(0-244$.

1994 : Découverte paléolithiques sur les Hauts de Garonne, in : L.Entre-DeuxMers à la recherche de son identité, Actes du $4^{\circ}$ colloque sur l'Entre-DeuxMers, 15-17 oct. 1993, Saint-Loubès, Lormont et Saint-Louis-de-Montforrand, p. 15-22.

I.NOIR M., TERrizIS

1979: Lames tronquées et usées du Magdalénien supérieur en Gironde, Bulletin de la Société préhistorique fran(aise, 76,1, p. 19-23.

I WOFF S.

1962 : La "serpette" du Magdalénien VI de Loubressac, commune de Mazcrolles (Vienne), nouveau fossile directeur régional lithique du département de la Vienne et de ses environs, Bulletin de la Soriété préhistorique fransaise. I.IX, 3-4, p. 278-288.

Misive A.

1940 : Découverte d'une grotte préhistorique à Bouliac, Procès verbaux de la 
Société linnéenne de Bordeaux, XCI, p. 37.

MAITESIN-F.LBRE G.

1946 : Sur l'âge de la groute de Haurets, Procès verbaux de la Société linnéenne de Bordeaux, XCIII, séance du 6 février, p. $188-192$.

\section{M.ARQI TET J.C.}

1984 : Paléoenvironnement et chronologie des sites du domaine atlantique francais d'âge pléistocène moyen et supérieur. d'apres l'etude des rongeurs, thèse de Doctorat d'État es sciences naturelles, 6.37 p.

\section{MIILTII D.}

1991 : Géomorphologie des Graves Sud du Bordelais, IER de géographic physique, LER de géographic et d'études régionales, univ de Bordeaux III, $186 \mathrm{p}$.

1992 : Les industries sur galets des Moyennes terrasses des Grazes Sud de Bordeaux. Contribution à l'étude du Paléolithique dans la basse vallee de la Garonne, DEA d'anthropologie : anthropologie des hommes fossiles et préhistoire, univ. de Bordeaux I. 104 p.

Milli:t F., Millek D.

1991 : Moyenne terrasse du vignoble des Graves de Bordeaux, Ciallia Informations, 1991-1, p. 109-110.

MoIsin L.

1978a: Recherches sur les terrasses alluviales du Libournais et leurs industries préhistoriques, thèse de Doctorat de l'univ. de Bordeaux, $421 \mathrm{p}$.

1978b: Les terrasses alluviales du Libournais. Interprétations chronologiques. Étude de leur contenu archéologique, Bulletin de la Société d'anthropologie du Sud-Ouest, XII, 4, p. $85-106$.

1979 : Les industries préhistoriques des terrasses alluviales du Libournais, Revue historique et archéologique du Libournais, XIVII, 172, p. 41-(62.

1986 : Quelques données sur le peuplement du bassin inférieur de la Dordogne au Paléolithique inférieur et moven, Bulletin de la Société d'an- thropologie du Sud-Ouest, XXXI, 2, p. 119-121.

1987: Les formations alluviales du bassin inférieur de la Dordogne et leurs industries paléolithiques, Paris, éd. du C.NRS, Cahiers du Quaternaire, 10, $423 \mathrm{p}$.

Ot\%RIT I.

1986 : Recherches sur les faumes du Dryas ancien en Gironde. Le gisement de SaintGermain-la-Rivière, thèse de Doctorat en géologie du Quaternaire, univ. de Bordeaux I, $147 \mathrm{p}$.

PASSINMARD E.

1944 : La carerne d'Isturitz en Pays basque, Préhistoire, IX, p. 7-84.

Rigit D J.-P., Le.NOIR M., DellPi(H F,

1972 : Fouilles de sauretage dans le gisement magdalénien de Fongaban, commune de Saint-Émilion (Gironde), L'Anthropologie, 76, 7-8, p. $595-629$.

\section{Rol'ssot A.}

1970 : Flûtes et sifflets paléolithiques en Gironde, Reoue historique de Bordeaux et du département de la Gironde, 19, noux: série, p. 512.

RoISSOT A., FHRrIER J.

1970) : Le Roc de Marcamps (Gironde). Quelques nouvelles observations, Bulletin de la Société préhistorique fransaise, 67, ét. et trav. 1, p. 293-303.

1971 : La grotte de Fontarnaud, commune de Lugasson (Gironde), Bulletin de la Société préhistorique fransaise, 68, ét. et trav: 2, p. 505-520.

ROLSSOI-LARROQLE J.

1979 : Stations aziliennes du Médoc et des Landes de la Gironde. Documents et problèmes, in: Sonnerille-Bordes D. de (éd.), La fin des Temps glaciaires en Europe. Chronostratigraphie et écologie des cultures du Paléolithique final, 1 , Colloques internationaux du C.NRS, $\mathrm{n}^{\circ}$ 271, Talence, 24-28 mai 1977, Paris, éd. du (.NRS, p. 387-400).

RotSSOT-L.ARROQL F. J., CALliEDE J.P. 1976 : Stations aziliennes du Médoc: le
Vieux port à lacanau et le Luc à Talais, et l'Azilien du Médoc et des landes de la Cironde. Bulletin de la Société d'anthropologie du Sud-(Ouest, XI, 4, p. 152-163.

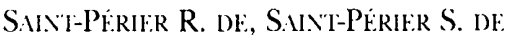
1952 : La grolle d'Isturitz -III- Les Solutréens, les Aurignaciens et les Moustériens, Paris, Masson, Archives de l'Institut de paléontologie humaine, mém. 25, 264 p.

SIRT:IX M., BORIDES F.

1972 : Le Moustérien de Chinchon (Gironde), Bulletin de la Société préhistoriques francaise, 69, et. et trav. 1, p. 324-336.

SI.OTT-MoII.t.R R.

1988 : Contribution à l'étude paléontologique d'un gisement préhistorique : l'exemple du Roc de Marcamps (Gironde), mémoire de DES, univ: de Bordeaux I, 173 p.

S.MITH P.

1966 : Le Solutrén en France, Publication de l'Institut de Préhistoire de l'université de Bordeaux, mém. os, 449 p.

SONNETILIF-BORDES D. DE

1965 : Deux gisements paléolithiques du Sud-Ouest de la France : le Trou de la Chère (Dordogne) et Pair-non-Pair (Gironde), I.Anthropologie, 69, 1-2, p. 169-175.

1988 : Les pointes à affinités nordiques dans le Paléolithique final au sud de la I.oire, in: Otte M. (éd.), De la Loire à l'Oder. Les civilisations du Paléolithique final dans le Vord-Ouest européen, Actes du colloque de Liège, déc. 1985, BAR, International Series, 444, p. 621-643.

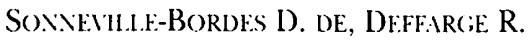

1974 : Lames retouchées magdaléniennes du Morin (Gironde), Tephyrus, XXV, p. 95-105.

Tíro A., LeNoir M.

1992: Gensac le Pigeonnier, Bilan scientifique, Direction régionale des affaires culturelles, Aquitaine. Service Régional de l'Archéologie, Bordeaux, p. 5.5. 
VINETT D.

1988 : Analyses polliniques de coprolithes dHyene provenamt des gisements sui- vants : Saint Hippolyte (Puy-de-Dôme), Chatelperron (Allier), La Chaise de Vouthon (Charente), Cónac (Gironde),
Camiar (Gironde), Bourdetle (Lot-et(iaronne), mémoire de DEA d'anthropologie, univ de Bordeaux I, $126 \mathrm{p}$. 